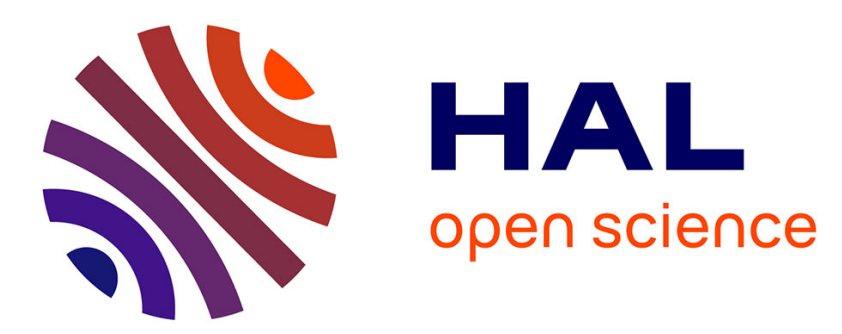

\title{
Formation et difficultés de fonctionnement des équipes successorales: une analyse fondée sur deux études de cas exploratoires et longitudinales
}

\author{
Catherine Thévenard-Puthod
}

\section{- To cite this version:}

Catherine Thévenard-Puthod. Formation et difficultés de fonctionnement des équipes successorales: une analyse fondée sur deux études de cas exploratoires et longitudinales. Management international, 2014, 18 (4), pp.131-150. 10.7202/1026034ar . hal-01132603

\section{HAL Id: hal-01132603 \\ https://hal.univ-grenoble-alpes.fr/hal-01132603}

Submitted on 9 Feb 2018

HAL is a multi-disciplinary open access archive for the deposit and dissemination of scientific research documents, whether they are published or not. The documents may come from teaching and research institutions in France or abroad, or from public or private research centers.
L'archive ouverte pluridisciplinaire HAL, est destinée au dépôt et à la diffusion de documents scientifiques de niveau recherche, publiés ou non, émanant des établissements d'enseignement et de recherche français ou étrangers, des laboratoires publics ou privés. 


\title{
Formation et difficultés de fonctionnement des équipes successorales : une analyse fondée sur deux études de cas exploratoires et longitudinales
}

\begin{abstract}
La transmission d'une entreprise à une équipe de successeurs est un sujet peu étudié en entrepreneuriat, que ce soit dans la littérature sur la transmission d'entre-prise ou dans celle consacrée aux équipes d'entrepreneurs. En se basant sur l'étude longitudinale de deux cas de successions, cet article apporte des connaissances sur le lien entre le mode de constitution de ces équipes et les difficultés qu'elles ren-contrent ensuite. Il met en relief l'influence forte du cédant sur le déroulement de ce type particulier de succession et recense ainsi trois facteurs qui s'avèrent être des sources de dysfonctionnements importants pour ces équipes.
\end{abstract}

Mots clés : succession familiale - équipe de successeurs - formation des équipes - parties prenantes - études de cas longitu-dinales

$\mathrm{D}$ epuis une vingtaine d'années, on assiste dans le domaine de l'entrepreneuriat à une croissance des recherches sur les équipes d'entrepreneurs. En effet, si on a pendant longtemps considéré que l'entrepreneur était un homme seul (Cooney, 2005), on reconnaît depuis lors que la création d'entreprise est souvent un phénomène collectif (Kamm et al, 1990; Cooper et Daily, 1997; Iacobbucci et Rosa, 2010; Schjoedt et al, 2013). La réunion de plusieurs personnes au sein d'une équipe d'entrepreneurs facilite ce processus sur de nombreux aspects (par exemple sur le plan des ressources et compétences amenées par les différents fondateurs) et est ainsi un gage non seulement de meilleure réussite du lancement de la nouvelle structure, mais aussi de sa croissance (Eisenhardt et Schoonhoven, 1990; Kamm et al, 1990; Cooney, 2005). Cependant, si des auteurs de plus en plus nombreux s'intéressent au rôle joué par les équipes 
d'entrepreneurs en situation de création ex nihilo, il existe à ce jour très peu de travaux dans le domaine de la transmission d'entreprise en général, et de la succession des PME familiales en particulier. Pourtant, la succession est une situation entrepreneuriale spécifique (Cadieux et Deschamps, 2009), dans la mesure où il ne s'agit pas de créer une structure sur-mesure correspondant aux désirs des créateurs, mais bien de diriger, de faire évoluer et de développer une structure existante, en tenant compte des aspirations de ses différentes parties prenantes internes et externes (Deschamps, 2002; Bornard et Thévenard-Puthod, 2009). En outre, cette situation entrepreneuriale mérite une attention particulière car un nombre significatif d'entreprises familiales ne survivent pas à leur changement de propriétaires ${ }^{1}$ (Mouline, 2000; Carlock et Ward, 2001; Bughin et al., 2010). Enfin, même si l'on ne dispose pas de statistiques fiables en la matière, certains chercheurs prédisent que le nombre d'équipes de successeurs va aller en s'accroissant (Cater et Justis, 2010; Farrington et al, 2012).

Cet article se propose par conséquent d'apporter des enseignements sur ce type particulier de transmission qu'est celle d'une petite entreprise familiale à une équipe de successeurs. Nous transposons pour cela la définition des équipes entrepreneuriales de Cooney (2005) au contexte de la succession en considérant que les équipes de successeurs (ou équipes successorales) sont constituées d'au moins deux individus à qui l'on transmet à la fois la propriété de l'entreprise familiale et le pouvoir de direction. Plus précisément, nous cherchons à mieux appréhender le processus de formation de ces équipes et nous souhaitons comprendre comment la façon dont ces équipes se constituent peut être une source de succès ou d'échec de ce mode particulier de succession. Face à ce questionnement, une méthodologie qualitative fondée sur des études de cas longitudinales est apparue pertinente. C'est pourquoi deux cas de transmission à une équipe de successeurs ont été analysés. Ils permettent notamment de mettre en exergue le rôle négatif que peut parfois jouer le cédant sur la réussite d'une équipe (alors même qu'il désire bien faire) lorsqu'il désigne luimême les membres de cette dernière. Ce mode de constitution peut introduire une trop grande hétérogénéité parmi les membres de l'équipe et aboutir à l'absence de liens forts les unissant, ce qui favorise l'émergence de nombreux conflits que les successeurs ne parviennent pas toujours à dépasser.

La première partie expose une synthèse des travaux issus de la littérature sur la succession familiale et les équipes entrepreneuriales. Elle débouche sur la proposition d'une grille d'analyse des transmissions à une équipe successorale

1. Handler (1994) indique ainsi, en reprenant les études de Beckard et Dyer (1983), que $30 \%$ des entreprises familiales survivent à leur transmission à la deuxième génération et $10 \%$ seulement à la troisième génération. Plus récemment, l'enquête Oséo Bdpme (2005) révèle qu'en moyenne plus d'une transmission sur cinq échoue avant six ans.

2. La définition généralement admise de l'entreprise familiale est la suivante : une entreprise dans laquelle au moins deux personnes d'une et se termine par la justification de nos choix méthodologiques. Dans la deuxième partie, nous présentons les résultats des analyses des deux études de cas réalisées. Enfin, dans une dernière partie, nous mettons en évidence les principaux enseignements de cette étude empirique, concernant la formation et le succès des équipes successorales.

\section{Une grille d'analyse issue de la littérature sur les équipes entrepreneuriales et adaptée au contexte de la succession}

\section{UN FAIBLE NOMBRE DE TRAVAUX SUR LA TRANSMISSION D'ENTREPRISE FAMILIALE À UNE ÉQUIPE}

Depuis près de 60 ans, de nombreux chercheurs se sont intéressés à l'épineux problème de la succession, en particulier dans le contexte des entreprises familiales ${ }^{2}$ (Christensen, 1953; Handler, 1994). Tous s'accordent à considérer la succession comme un processus complexe et itératif (Cater et Justis, 2009), composé, selon les auteurs, de trois à sept phases qui aboutissent au transfert de la propriété et de la direction de l'entreprise (Le Breton et al, 2004; Lambrecht, 2005). Pour Cadieux (2007) par exemple, le processus démarre bien avant l'entrée effective du successeur dans l'entreprise, par une phase d'initiation. Au cours de son enfance et de sa jeunesse, le futur successeur se façonne une image de l'entreprise et du rôle de chef d'entreprise (Cruz et al, 2013), par son interaction quotidienne avec ses parents et d'éventuels stages ou emplois d'été au sein de l'entreprise familiale. La phase d'intégration est ensuite une phase au cours de laquelle le successeur est recruté dans l'entreprise pour se familiariser davantage avec celle-ci et se forger progressivement un savoir-faire (Hawkins, 2011). Puis vient la phase de règne-conjoint pendant laquelle il occupe une position plus officielle de successeur et où un véritable transfert de responsabilité et d'autorité s'opère avec le cédant. L'entreprise peut ainsi être gérée pendant un temps plus ou moins long par une équipe managériale trans-générationnelle composée du parent cédant et du successeur (Cater et Justis, 2010; Sciascia et al, 2013). Une fois que le successeur a acquis les compétences nécessaires et a réussi à faire ses preuves, vient alors la phase de désengagement du cédant. Ce dernier se retire de la direction de l'entreprise, mais il peut cependant continuer à s'impliquer dans l'activité, en assumant différents rôles plus ou moins symboliques tels que celui d'administrateur, de garde-fou incarnant les valeurs de l'entreprise, d'intermédiaire avec les clients et fournisseurs clés ou encore de support technique et de consultant (Cadieux, 2007).

\footnotetext{
même famille détiennent au minimum $51 \%$ du capital et exercent une influence considérable sur l'activité en occupant des postes clés de management (Farrington et al, 2012). Certains chercheurs rajoutent à ces deux critères l'intention de continuité par le transfert à au moins un des membres de la famille (Cadieux et Brouard, 2009). Toutefois ce critère est relativement subjectif et ne peut pas toujours être évalué ex ante. En effet les successeurs manifestent parfois tardivement leur désir de reprendre l'entreprise familiale.
} 
Les chercheurs ont également pu identifier un très grand nombre de facteurs de succès de ce processus (Le Breton-Miller et al, 2004). Ainsi, il a tout d'abord été mis en avant que ce dernier devait être planifié à l'avance pour une meilleure réussite et qu'une bonne communication entre les acteurs était essentielle. Un timing adéquat, qui laisse notamment suffisamment de temps pour former le successeur, a été régulièrement relevé (Dyck et al, 2002). Les caractéristiques du fondateur ont également reçu beaucoup d'attention, dans la mesure où cet acteur est considéré comme le personnage central de la succession. Sa personnalité, sa motivation (Handler, 1994) et sa capacité à gérer la transition de rôle (Cadieux et Deschamps, 2011) ont été régulièrement soulignés par les auteurs. Puis plus récemment, les chercheurs se sont intéressés aux caractéristiques du successeur telles que sa motivation, son engagement (Cabrera-Suarez, 2005), ou encore la qualité de la relation entretenue avec le prédécesseur (Venter et al, 2005; Cater et Justis, 2009). Des facteurs de contexte comme l'harmonie familiale ou la santé de l'entreprise transmise ont enfin été soulignés (De Massis et al, 2008).

Toutefois, dans la très grande majorité de ces recherches, la transmission de l'entreprise se réalise (au moins implicitement) au profit d'un seul successeur, héritier(e) du prédécesseur. Très peu de chercheurs se sont intéressés à la spécificité des équipes de successeurs, même si certains soulignent qu'elles peuvent être source d'une plus grande performance pour l'entreprise et que ce type de succession est amené à se développer (Aronoff, 1998; Cater et Justis, 2010; Farrington et al, 2012). Afin de mieux analyser le phénomène, il convient donc d'aller puiser dans le champ plus large de l'entrepreneuriat pour retirer des enseignements sur la formation et le fonctionnement de ces équipes.

\section{LES MODES DE CONSTITUTION DES ÉQUIPES} ENTREPRENEURIALES ET LEUR EFFICACITÉ

Dans la littérature en entrepreneuriat, on recense plusieurs motivations des protagonistes à former une équipe. On trouve tout d'abord le fait de vouloir bénéficier d'une plus grande diversité de connaissances et de savoir-faire (Naffakhi et al, 2008). La complexité des compétences nécessaires à la création d'entreprise exige en effet souvent de rapprocher des savoir-faire complémentaires (Harrison et Leitch, 1995; West, 2007; Ucbasaran, 2008). L'association facilite également l'obtention de ressources financières supplémentaires, par la mobilisation des réseaux sociaux des partenaires (Ucbasaran et al., 2003; Chen et Wang, 2008). Elle permet par ailleurs de contourner les limites en temps et en attention d'un dirigeant qui agirait seul (Iacobbucci et Rosa, 2010) et introduit une spécialisation des tâches qui peut s'avérer fort opportune devant la masse de travail à fournir. D'un point de vue plus psychologique, enfin, la constitution d'une équipe autorise une meilleure répartition des risques (Almeida et Wolfenzon, 2006) et diminue l'anxiété des individus (Harisson et Leitch, 1995). Elle abaisse la réticence des personnes à se lancer dans l'aventure créative et les incite au passage à l'acte (Moreau, 2006).

Les chercheurs ayant travaillé sur la formation des équipes entrepreneuriales distinguent ensuite deux types de processus : soit un individu a une idée de création d'entreprise et convainc les autres de se joindre à lui, soit l'équipe préexiste et cherche ensuite une idée (Kamm et Nurick, 1993; Francis et Sandberg, 2000). Le mode de sélection des membres d'une équipe peut ainsi s'opérer selon deux approches (Moreau, 2006; Chabaud et Condor, 2009; Ben-Hafaiedh-Dridi, 2010) : une approche stratégique qui trouve ses fondements théoriques dans la ResourceBased View (Barney, 1991; Grant, 1991) ou la Théorie de la Dépendance des Ressources (Pfeffer et Salancik, 1978) et une approche sociale reposant sur la Théorie des Réseaux Sociaux (Granovetter, 1985; Burt, 1992). L'approche stratégique correspond à une vision instrumentale et rationnelle du processus de formation d'une équipe. Elle considère qu'un entrepreneur leader va former une équipe une fois qu'il a identifié les ressources nécessaires à son projet et sélectionner des membres capables de lui amener ces ressources et compétences critiques. Dans l'approche sociale, l'entrepreneur leader va plutôt sélectionner des individus dont il se sent proche et qui font partie de son réseau social. Une certaine homophilie, fondée sur des caractéristiques personnelles telles que l'âge, le genre et l'origine ethnique, ou sur des attitudes et valeurs identiques, est alors recherchée. Les travaux empiriques disponibles montrent que les équipes émergent plutôt suivant la seconde approche, c'est-à-dire à partir de liens familiaux (Cruz et al, 2013), de considérations d'amitié ou de relations préexistantes (Kamm et al, 1990; Francis et Sandberg, 2000; Moreau, 2006). Toutefois certains auteurs s'accordent à considérer qu'un mélange des deux modes de sélection serait une situation idéale (Moreau, 2006), aboutissant ainsi à la création d'une «dream team» (Chabaud et Condor, 2009). En effet, la complémentarité des ressources et compétences permet de couvrir les différents besoins liés à la création d'entreprise (Francis et Sandberg, 2000). En complément, des liens forts permettent d'éviter la présence d'objectifs contradictoires, d'attitude envers le travail et de valeurs différentes (Timmons, 1979; Kotkin, 1986; Francis et Sandberg, 2000; Cruz et al, 2013; Lung et al, 2013) ou d'incompatibilités de personnalités (West, 2007) qui sont autant de sources potentielles de conflits pouvant mettre à mal le bon fonctionnement d'une équipe. En se faisant confiance au préalable (Chen et Wang, 2008), les membres de l'équipe gagnent du temps en coordination et peuvent se concentrer plus rapidement sur la résolution de problèmes concrets liés à la création d'entreprise (Eisenhardt et Schoonhoven, 1990).

Enfin, la présence d'un leader est également soulignée dans plusieurs études empiriques portant sur les critères d'efficacité de ces équipes (Timmons, 1994; Ensley et al, 2000). Celui-ci ne possède pas toujours des compétences ou des connaissances supérieures aux autres membres mais 
est doté d'une vision stratégique plus aigüe. Persuasif et déterminé, il influence les interactions et propage cette vision au sein de l'équipe (Harper, 2008).

Certains chercheurs estiment que ces enseignements issus de la littérature sur le fonctionnement des équipes peuvent s'appliquer à de très nombreux contextes. Ainsi Farrington et al (2012) ont très récemment mené une étude quantitative sur la transmission des entreprises familiales à un type d'équipe : les fratries. Leurs résultats mettent notamment en avant deux facteurs de succès déjà identifiés par la littérature sur les équipes : la présence d'un leader visionnaire orienté sur le long terme et l'hétérogénéité des compétences des membres de la fratrie. Il existe cependant à nos yeux quelques différences entre les équipes entrepreneuriales (au sens de celles qui réalisent une création ex nihilo) et les équipes successorales qui méritent d'être soulignées, car elles pourraient influencer la formation et le fonctionnement de ces dernières. En effet, dans le cas d'une création ex nihilo, les entrepreneurs créent une structure surmesure, en fonction de leurs aspirations propres et de leurs compétences, tout en tenant compte, bien entendu, des possibilités offertes par l'environnement (soutiens financiers, prédisposition des clients à accepter la nouvelle offre...). Le cas de la succession est différent dans la mesure où il s'agit tout d'abord de convaincre le propriétaire actuel de céder sa structure. Ensuite, les successeurs doivent diriger une structure qui a été créée par une ou plusieurs autres personnes et qui fonctionne déjà avec ses propres routines. Ses différentes parties prenantes internes et externes (famille, salariés, clients, fournisseurs...) peuvent être très attachées à l'ancienne direction et à la préservation des valeurs et du mode de fonctionnement déjà en place (Deschamps, 2002; Bornard et Thévenard-Puthod, 2009). La problématique est davantage celle de la gestion du changement, qui soulève des difficultés particulières, notamment au niveau de l'influence forte que peuvent opérer ces différentes parties prenantes sur le fonctionnement de l'équipe et sur le déroulement de la succession. On ne peut donc pas, dans un processus de succession familiale, uniquement se focaliser sur l'équipe de successeurs. Il faut également tenir compte de celle du/des cédant(s) et des éventuelles autres parties prenantes de l'entreprise.

En définitive, compte tenu du faible nombre de recherches portant sur les équipes successorales et des différences de contexte existant entre la création ex nihilo et la succession, les questions de recherche qui ont guidé ce travail et structuré notre cadre d'analyse (cf. figure $\mathrm{n}^{\circ} 1$ ) sont au nombre de deux :

- Q1 : Pourquoi et comment les équipes successorales se forment-elles?

- L'idée de reprise est-elle préalable à la constitution de l'équipe ou bien est-ce l'inverse? Quelles sont les motivations non seulement des successeurs, mais aussi des cédants à se lancer dans une telle forme de succession? Quels sont les modes de sélection des membres utilisés pour constituer l'équipe successorale?

- Q2 : Comment ce mode de constitution influence-t-il le fonctionnement des équipes?

- Quelles difficultés rencontrent-elles ? En quoi la façon dont ces équipes se constituent peut-elle faciliter leur réussite ou au contraire constituer des obstacles majeurs?

\section{UNE MÉTHODOLOGIE QUALITATIVE FONDÉE SUR DEUX ÉTUDES DE CAS LONGITUDINALES}

Comme le sujet de la transmission d'une PME familiale à une équipe de successeurs a reçu jusque-là peu d'attention des chercheurs, une approche exploratoire a été estimée préférable (Kamm et al, 1990; Yin, 1994; Creswell, 2009). Les questions de recherches incluant la prise en compte de plusieurs acteurs (cédants et successeurs notamment), les études de cas ont été considérées comme la méthode d'accès

\section{FIGURE 1}

\section{Le cadre d'analyse retenu}

Q1 - Mode de constitution de l'équipe successorale :

- Type de processus de formation (antériorité du projet de reprise par rapport à la constitution de l'équipe)

- Motivation des successeurs à former une équipe

- Motivations des cédants à transmettre à une équipe

- Critères de sélection des membres de l'équipe (complémentarité des ressources et compétences, proximité sociale)

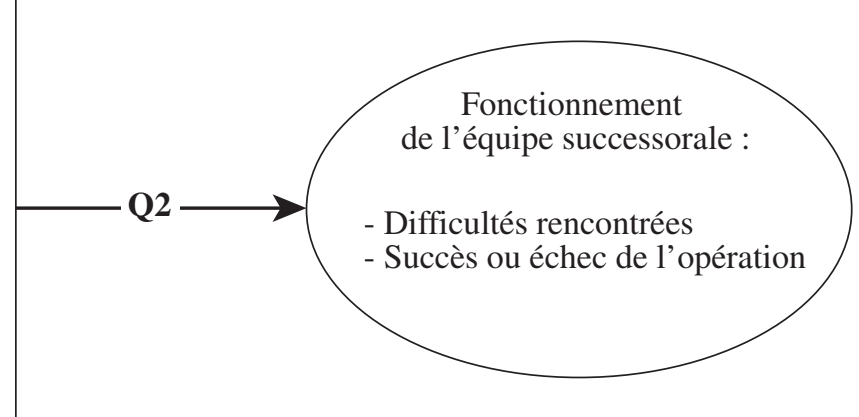


aux données la plus pertinente (Wacheux, 1996) ${ }^{3}$. En outre, étant donné que la succession est un processus qui évolue dans le temps, parfois sur une longue période, une approche longitudinale était requise (Iacobbucci et Rosa, 2010).

Comme le souligne Wacheux (1996, p. 95), «le nombre de cas dépend des objectifs de la recherche. S'il s'agit d'explorer des pratiques nouvelles, ou discuter un questionnement original, un ou quelques cas suffisent pour rentrer dans une logique de découverte». Nous avons ainsi choisi d'étudier en profondeur deux cas de succession. La compréhension d'un phénomène dépendant du choix des cas (Stake, 1994), ces deux cas ont été sélectionnés pour des motifs théoriques, sur la base de deux critères d'homogénéité (tableau $\mathrm{n}^{\circ} 1$ ) : il s'agit non seulement de cas de transmissions d'entreprises familiales à une équipe de successeurs, mais ils partagent comme autre point commun de réunir des successeurs de types différents. En effet, on trouve dans les deux opérations étudiées une équipe constituée à la fois d'enfants du cédant et de salariés de l'entreprise. Au niveau des critères d'hétérogénéité, ces cas diffèrent en termes de taille et de métiers de l'entreprise (mécanique et menuiserie), ainsi que de taille de l'équipe de successeurs (quatre membres d'un côté, deux de l'autre).

Dans le premier cas (Mécanix), le processus de succession a duré six ans ${ }^{4}$ (2002-2007). Nous avons débuté le recueil de données seulement au début de l'année 2004, lorsque l'équipe potentielle de successeurs a été «désignée» et que le montage financier a été arrêté. Nous avons suivi cette succession jusqu'au départ d'un des membres de l'équipe de successeurs et l'acquisition finale de la propriété de l'entreprise par les trois autres. Pour le second cas (Menuibois), le processus a duré cinq ans. Il a également

\section{TABLEAU 1}

\section{Présentation des deux cas étudiés et du recueil de données effectué}

\begin{tabular}{|c|c|c|}
\hline CAS & Mécanix & Menuibois \\
\hline Activité & $\begin{array}{l}\text { Mécanique } \\
\text { (rectification et usinage mécanique) }\end{array}$ & Menuiserie \\
\hline Date de création & 1979 & 1979 \\
\hline Date(s) officielle (s) de la succession & $13 / 12 / 2007$ & $\begin{array}{c}1 / 10 / 2004 \\
\text { puis } 12 / 12 / 2006\end{array}$ \\
\hline $\begin{array}{l}\text { Nb de salariés au début du processus } \\
\text { de succession }\end{array}$ & 22 & 1 \\
\hline CA au début du processus de succession & 1,5 million d'euros & $200000 €$ \\
\hline $\begin{array}{c}\text { Composition initiale de l'équipe } \\
\text { de successeurs }\end{array}$ & Fille du cédant +3 salariés & Fils du cédant +1 salarié \\
\hline Période de suivi du cas & $\begin{array}{l}\text { Février 2004- Décembre } 2007 \\
\text { (puis 2012) }\end{array}$ & $\begin{array}{l}\text { Février 2004- Février } 2008 \\
\text { (puis 2012) }\end{array}$ \\
\hline Entretiens réalisés & $\begin{array}{c}\text { Février } 2004 \text { : cédant } \\
\text { Mars } 2004: \text { successeurs } \\
\text { Juin } 2004: \text { cédant + consultant } \\
\text { Décembre } 2004: \text { successeurs } \\
\text { Mars } 2005: \text { cédant } \\
\text { Novembre } 2005 \text { : successeurs } \\
\text { Juillet } 2006: \text { successeurs } \\
\text { Décembre } 2006: \text { consultant } \\
\text { Janvier } 2007: \text { successeurs } \\
\text { Décembre } 2007: \text { successeurs }\end{array}$ & $\begin{array}{l}\text { Février } 2004: \text { cédant } \\
\text { Novembre } 2004: \text { successeurs } \\
\text { Juin } 2005: \text { successeurs } \\
\text { Décembre } 2005: \text { successeurs } \\
\text { Septembre } 2006: \text { cédant } \\
\text { Janvier } 2007: \text { successeurs } \\
\text { Février } 2008: \text { cédant }\end{array}$ \\
\hline
\end{tabular}

3. Les études de cas permettent en effet de procéder à «une analyse spatiale et temporelle d'un phénomène complexe par les conditions, les événements, les acteurs et les implications» (Wacheux, 1996, p. 89).

4. Il est en effet illusoire de pouvoir dater avec précision le début réel d'un processus de succession, en particulier quand celui-ci met en jeu un enfant du cédant qui, de façon implicite, a été impliqué dans le processus bien avant que son nom ne soit évoqué de façon officielle en tant que successeur de l'entreprise (Cadieux et Brouard, 2009). Nous avons donc considéré que le processus débutait lorsque le cédant a délibérément évoqué la succession avec ses successeurs potentiels et entrepris des démarches concrètes en ce sens. En outre, en suivant les recommandations de Chandler et Hanks (1998) et de Francis et Sandberg (2000), nous avons inclus dans l'équipe de successeurs les membres qui l'ont rejointe pendant les deux premières années du processus «officiel» de succession. 
débuté en 2002, mais n'a là-aussi été suivi qu'à partir de 2004, juste quelques mois avant la première répartition des parts du capital de l'entreprise. Il a été étudié jusqu'en 2008, soit un peu plus d'un an après la reprise définitive par un seul des membres de l'équipe de départ. Nous avons également vérifié qu'en 2012 ces deux entreprises étaient toujours aux mains des mêmes successeurs.

Nous avons utilisé plusieurs sources d'informations pour analyser ces cas. Une trentaine d'entretiens semidirectifs ont été menés (de 2004 à 2008) auprès des principaux protagonistes impliqués dans l'opération (cédants, successeurs, mais aussi un consultant externe). Le guide d'entretien a été construit sur la base des questions de recherche définies précédemment. Chacune des successions a été suivie à un rythme semestriel (tableau $\mathrm{n}^{\circ} 1$ ). A chaque nouvel entretien, nous faisions le point sur l'évolution du projet (les étapes franchies, les événements clés, les changements d'organisation de l'équipe, les difficultés rencontrées et les étapes à venir) et sur le vécu des acteurs (l'évolution de leurs motivations par exemple ou les réactions des différentes parties prenantes). Dans un effort de triangulation des données (Yin, 1994), nous avons eu accès à des sources d'information secondaires comme les contrats de vente ou les documents supports des montages financiers. Nous avons également exercé une observation passive puisque nos entretiens ont tous eu lieu dans les locaux des entreprises transmises, ce qui nous a permis de suivre les transformations progressives de ces dernières et de pouvoir observer in vivo les liens existants entre les protagonistes (et leur détérioration).

Pour coder les données, nous avons utilisé une procédure d'analyse de contenu (Strauss, 1987). Pour l'analyse intra-site, nous avons codé les données en thèmes correspondant aux différentes composantes de notre cadre d'analyse (motivation des cédants à la transmission, motivations des cédants et successeurs à la transmission/succession en équipe, préparation des acteurs, nature des liens unissant les membres de l'équipe, profil des successeurs et degré de complémentarité, modalité de constitution de l'équipe...). Nous avons également élaboré des relevés d'événements chronologiques pour synthétiser les étapes clés du processus de succession et pour mieux identifier les facteurs d'échec de ces processus (Huberman et Miles, 1991). Nous avons ensuite procédé à une analyse transversale pour faire émerger des patterns et tenter de comprendre les facteurs à l'origine des difficultés rencontrées, en utilisant des matrices destinées à faciliter la comparaison des deux cas (Huberman et Miles, 1991).

Par ailleurs, les critères de réussite que nous avons retenus pour évaluer les successions ont été les suivants : l'entreprise devait avoir été réellement transmise, à l'équipe initialement constituée (les successeurs devaient contrôler le capital et diriger l'entreprise; Handler, 1994), et être suffisamment en bonne santé pour assurer sa pérennité (Le Breton et al, 2004; Venter et al, 2005).

\section{L’analyse du déroulement des deux successions étudiées}

Du seul point de vue de la transmission, celles-ci peuvent dans un premier temps être assimilées à des succès. En effet, la vente des deux entreprises a bien été réalisée et en 2012 (soit quatre à cinq ans après les opérations), ces entreprises existent toujours et on peut être rassuré sur leur pérennité, au moins à moyen terme. Cependant du point de vue de la transmission à une équipe de successeurs, les résultats sont plus décevants. La mise en place de ces équipes successorales, à l'instar de certaines équipes de créateurs d'entreprises, s'est en effet réalisée de façon chaotique et leur composition n'est pas demeurée stable dans le temps. Les deux cas étudiés se sont ainsi soldés par la sortie de membres composant initialement l'équipe de successeurs. Dans le cas Mécanix, c'est la fille du cédant qui est partie, laissant les trois anciens salariés reprendre l'entreprise. L'entreprise n'est donc plus aujourd'hui familiale. Dans le cas Menuibois, c'est le salarié qui a fini par abandonner le processus successoral, laissant le fils du cédant assumer seul ce projet. Ce dernier cas s'est donc au final transformé en succession «simple». Les principaux patterns qui ressortent de ces cas sont présentés dans les paragraphes qui suivent et sont illustrés de verbatim.

\section{UNE VOLONTÉ DE TRANSMETTRE DES CÉDANTS FORTE MAIS UNE SUCCESSION PEU PLANIFIÉE}

Dans les deux cas, la succession n'avait pas été réellement planifiée longtemps à l'avance. Même si implicitement les cédants y pensaient depuis un certain temps, ils n'avaient rien mis en œuvre de concret pour démarrer le processus. C'est l'approche de l'âge de la retraite qui leur a fait prendre conscience de la nécessité d'entamer les démarches. Ils ont alors affiché une volonté forte à ce que leur succession soit une réussite. Comme le déclare C.M., le cédant de Mécanix en début du processus : «ce n'est pas eux (les salariés) qui doivent faire le premier pas pour la transmission. C'est d'abord le chef d'entreprise qui doit avoir répondu à une question très importante : suis-je prêt, du jour au lendemain, à remettre les clefs, le chéquier et la carte bleue de l'entreprise au repreneur et à ne plus remettre les pieds dans l'entreprise? C'est une décision difficile à prendre mais c'est seulement quand le dirigeant est certain qu'il est capable de le faire que la démarche, à son initiative, peut débuter. (...) Transmettre à mes salariés, c'est pour moi plus motivant que prendre seulement un gros chèque pour partir aux Caraïbes $^{5} »$. Chez Menuibois, G.B. assure : «j'ai toujours fait en sorte de maintenir mon entreprise à niveau, notamment en matière de normes pour pouvoir la transmettre

5. Tous les passages en italique sont des citations des acteurs interrogés. 


\section{TABLEAU 2}

\section{Relevé des éléments chronologiques relatifs à la constitution de l'équipe successorale et du déroulement de la transmission du cas Mecanix}

\begin{tabular}{|c|c|c|c|c|}
\hline Dates & Phase $^{6}$ & Déroulement de la phase & $\begin{array}{c}\begin{array}{c}\text { Rôle } \\
\text { du } \\
\text { prédécesseur }\end{array} \\
\end{array}$ & $\begin{array}{c}\text { Rôle } \\
\text { des successeurs }\end{array}$ \\
\hline \multirow[t]{2}{*}{ Juin 2002} & \multirow{4}{*}{$\begin{array}{l}\text { Début du } \\
\text { processus : } \\
\text { constitution } \\
\text { de l'équipe de } \\
\text { successeurs }\end{array}$} & $\begin{array}{l}\text { Devant l'âpreté des négociations lors d'une réu- } \\
\text { nion consacrée à l'application de la loi sur les } 35 \\
\text { heures, C.M. lance à ses salariés «j'ai l'intention } \\
\text { de me retirer, la retraite est proche, si vous voulez } \\
\text { l'entreprise, je vous la laisse. Réfléchissez-y». Mais } \\
\text { en fait, l'idée lui trottait déjà dans la tête depuis plu- } \\
\text { sieurs mois du fait de son âge avancé. }\end{array}$ & $\begin{array}{c}\text { Initiateur } \\
\text { du processus }\end{array}$ & - \\
\hline & & $\begin{array}{c}\text { Première proposition à des salariés : C.M. convoque } \\
\text { les deux salariés les plus anciens dans l'entreprise } \\
\text { (âgés de } 46 \text { et } 52 \text { ans) pour leur proposer son affaire. } \\
\text { Ceux-ci refusent la proposition pour des raisons } \\
\text { d'âge, pour l'un, et de situation familiale, } \\
\text { pour l'autre. }\end{array}$ & $\begin{array}{c}\text { Initiateur du } \\
\text { processus et } \\
\text { sélectionneur } \\
\text { des membres } \\
\text { de l'équipe }\end{array}$ & - \\
\hline Juillet 2002 & & $\begin{array}{c}\text { Seconde proposition à } 3 \text { autres salariés : } \\
\text { ceux-ci ne s'y étaient pas préparés mais semblent } \\
\text { favorables à une reprise à } 3\end{array}$ & Sélectionneur & $\begin{array}{l}\text { Salariés qui } \\
\text { restent à leur } \\
\text { poste }\end{array}$ \\
\hline Aout 2002 & & $\begin{array}{l}\text { G.M. aborde de nouveau l'idée de projet de transmis- } \\
\text { sion avec ses } 3 \text { salariés, mais ceux-ci n'ont attaqué } \\
\text { aucune démarche et déclarent être freinés par l'ab- } \\
\text { sence de ressource financière à investir dans le projet. }\end{array}$ & Mobilisateur & $\begin{array}{l}\text { Salariés qui } \\
\text { restent à leur } \\
\text { poste; Attentisme }\end{array}$ \\
\hline $\begin{array}{l}\text { Décembre } \\
2002\end{array}$ & $\begin{array}{l}\text { Intégration } \\
\text { d'un nouveau } \\
\text { successeur }\end{array}$ & $\begin{array}{l}\text { U.M. (femme du cédant) prend sa retraite. } \\
\text { Recrutement de G.M. pour la remplacer au poste } \\
\text { d'attachée de direction. Décision de l'inclure dans } \\
\text { l'équipe de successeurs. }\end{array}$ & Sélectionneur & $\begin{array}{l}\text { Salariés qui } \\
\text { restent à leur } \\
\text { poste }\end{array}$ \\
\hline $\begin{array}{l}\text { Mars } 2003 \\
\text { (à janvier } \\
\text { 2004) }\end{array}$ & $\begin{array}{l}\text { Montage juri- } \\
\text { dico-financier }\end{array}$ & $\begin{array}{l}\text { C.M. prend RDV avec } 3 \text { conseillers juridiques pour } \\
\text { réfléchir au montage juridico-financier de la transmis- } \\
\text { sion. Trois scénarios sont élaborés, avec des niveaux } \\
\text { d'implication financière différents des successeurs. }\end{array}$ & Mobilisateur & $\begin{array}{l}\text { Salariés qui } \\
\text { restent à leur } \\
\text { poste; Attentisme }\end{array}$ \\
\hline Mai 2003 & $\begin{array}{l}\text { Sollicitation } \\
\text { d'un consul- } \\
\text { tant extérieur } \\
\\
\text { et transfert } \\
\text { progressif du } \\
\text { pouvoir de } \\
\text { direction }\end{array}$ & $\begin{array}{l}\text { C.M. et } \mathrm{S} \text { font appel à un consultant extérieur pour } \\
\text { les aider à développer l'entreprise. Constitution d'un } \\
\text { comité de direction où les } 4 \text { successeurs prennent } \\
\text { place au côté de CM. Des réunions régulières } \\
\text { (jusqu'en décembre 2005) sont organisées avec le } \\
\text { comité de direction pour réfléchir aux axes de déve- } \\
\text { loppement envisageables. G.M. souhaite tout mettre } \\
\text { en œuvre pour développer l'entreprise, les trois autres } \\
\text { successeurs optent davantage pour le statu quo. }\end{array}$ & $\begin{array}{c}\text { Manager } \\
\text { Mobilisateur }\end{array}$ & $\begin{array}{l}\text { Assistants. } \\
\text { Membres } \\
\text { du comité de } \\
\text { direction }\end{array}$ \\
\hline
\end{tabular}

6. Nous avons, dans un premier temps, souhaité reprendre les quatre phases des successions familiales identifiées dans la littérature (Cadieux et Brouard, 2009), mais cela s'est avéré difficile du fait de l'absence de réelle planification du processus et de la présence de plusieurs types de successeurs (familiaux et non familiaux). Les phases présentées ici sont donc davantage liées au contenu de chacune des étapes clés que l'on a pu distinguer dans nos deux cas. 


\begin{tabular}{|c|c|c|c|c|}
\hline Avril 2004 & $\begin{array}{l}\text { Début du } \\
\text { transfert de } \\
\text { propriété et } \\
\text { retrait définitif } \\
\text { du cédant des } \\
\text { fonctions de } \\
\text { direction }\end{array}$ & $\begin{array}{l}\text { Accord sur le montage financier. Création d'une } \\
\text { holding dans laquelle les } 4 \text { successeurs sont action- } \\
\text { naires égalitaires (à hauteur de } 20 \% \text { en tout : soit } 5 \% \\
\text { chacun) à côté de C.M. (actionnaire principal à } 80 \% \text { ). } \\
\text { La holding va prendre le contrôle de la SAS via un } \\
\text { LBO. Les successeurs restent salariés + touchent une } \\
\text { partie du résultat (rté actuelle : } 10 \% \text { ). Grâce à cette } \\
\text { dernière, ils pourront racheter à terme la totalité de la } \\
\text { holding (25\% chacun). Plan à } 3 \text { ans. } \\
\text { Prix de vente de la SAS : } 6 \text { millions d'euros }\end{array}$ & $\begin{array}{l}\text { Désengage- } \\
\text { ment }\end{array}$ & \multirow{7}{*}{$\begin{array}{l}\text { Managers } \\
\text { salariés }\end{array}$} \\
\hline $\begin{array}{l}\text { Avril } 2004- \\
\text { Août } 2004\end{array}$ & $\begin{array}{l}\text { Lutte pour le } \\
\text { leadership }\end{array}$ & $\begin{array}{l}\text { Petit à petit, un tandem apparaît pour le leadership } \\
\text { de l'équipe : la fille du cédant, G.M., et le salarié } \\
\text { R. Ils cherchent tous deux à s'imposer en tant que } \\
\text { dirigeants de la structure, mais avec des intérêts dif- } \\
\text { férents du fait de leur fonction et de leur formation } \\
\text { initiale (vision stratégique et financière pour G.M. } \\
\text { contre vision technique pour R.; vision à long terme } \\
\text { pour G.M. contre gestion plus quotidienne pour R.). }\end{array}$ & \multirow{6}{*}{$\begin{array}{c}\text { Mise en } \\
\text { situation des } \\
\text { successeurs } \\
\text { pour faire } \\
\text { émerger un } \\
\text { leader. Passe } \\
\text { dans l'entre- } \\
\text { prise une fois } \\
\text { par mois pour } \\
\text { voir si tout va } \\
\text { bien. Reste } \\
\text { en contact } \\
\text { régulier avec } \\
\text { sa fille pour la } \\
\text { conseiller. } \\
\text { Observateur, } \\
\text { consultant }\end{array}$} & \\
\hline $\begin{array}{l}\text { Septembre } \\
2004\end{array}$ & $\begin{array}{l}\text { Choix d'un } \\
\text { leader «offi- } \\
\quad \text { ciel» }\end{array}$ & $\begin{array}{l}\text { G.M. est finalement choisie pour occuper la fonction } \\
\text { de dirigeante de la SAS, les trois autres successeurs } \\
\text { demeurent membre du comité de direction }\end{array}$ & & \\
\hline Janvier 2005 & \multirow{4}{*}{$\begin{array}{l}\text { Conflits cogni- } \\
\text { tifs et affectifs }\end{array}$} & $\begin{array}{c}\text { Conflit important sur la définition des projets de } \\
\text { développement }\end{array}$ & & \\
\hline $\begin{array}{l}\text { Octobre } \\
2005\end{array}$ & & Remise en cause du mode de management de G.M. & & \\
\hline Février 2006 & & $\begin{array}{l}\text { Défiance des } 3 \text { successeurs salariés envers G.M. Ils } \\
\text { l'accusent de masquer les données financières }\end{array}$ & & \\
\hline $\begin{array}{l}\text { Septembre } \\
2007\end{array}$ & & $\begin{array}{c}\text { Nouveau désaccord important sur un projet de déve- } \\
\text { loppement }\end{array}$ & & \\
\hline $\begin{array}{l}\text { Novembre } \\
2007\end{array}$ & $\begin{array}{l}\text { Départ d'un } \\
\text { des membres } \\
\text { de l'équipe }\end{array}$ & $\begin{array}{l}\text { G.M. décide de quitter l'entreprise. Ce sont les } 3 \\
\text { salariés qui acquièrent finalement la propriété de } \\
\text { l'entreprise, R. devenant le nouveau dirigeant de la } \\
\text { structure. }\end{array}$ & Retrait & $\begin{array}{l}\text { Managers } \\
\text { propriétaires }\end{array}$ \\
\hline
\end{tabular}

facilement. Je me suis investi dans cette entreprise pendant plus de 25 ans, ce n'est pas pour la laisser mourir».

La succession s'est ensuite déroulée en plusieurs étapes, mais celles-ci n'ont pas toujours été aussi marquées que la littérature sur les successions familiales le laissait supposer (Cadieux et Brouard, 2009). L'initiation de certains successeurs a en effet été limitée, l'intégration s'est parfois superposée avec le règne-conjoint, qui a pu lui-même être très court (cas Mécanix), du fait de problèmes de santé du cédant. Aucun des successeurs ne s'était véritablement préparé à la succession avant le démarrage de celle-ci (les salariés ne s'attendaient pas à ce que leur dirigeant leur propose de reprendre l'entreprise et les enfants se destinaient, au départ, à d'autres carrières professionnelles). Les tableaux $\mathrm{n}^{\circ} 2$ et 3 présentent ainsi le déroulement chronologique des deux successions étudiées.

\section{DES ÉQUiPES CONSTITUÉES PAR LE CÉDANT POUR LA SUCCESSION}

En ce qui concerne la façon dont les équipes se sont constituées, on peut tout d'abord noter un point de convergence fort de ces deux cas : c'est la succession qui a déclenché la constitution de l'équipe. L'entreprise existant déjà et n'étant pas à créer, c'est le souhait du cédant de transmettre qui amorce le processus de succession et la constitution de l'équipe de successeurs. On peut donc parler d'équipes $a d$ hoc. Les motivations évoquées par les successeurs pour accepter de constituer une équipe sont plurielles et diffèrent selon les cas (cf. tableau $\left.{ }^{\circ} 4\right)$.

On retrouve toutefois des finalités comparables à celles identifiées en création ex nihilo. Ainsi le partage des tâches à réaliser à la direction de l'entreprise demeure une des raisons centrales dans les deux successions. Le cas Mécanix 


\section{TABLEAU 3}

\section{Relevé des éléments chronologiques relatifs à la constitution de l'équipe successorale et du déroulement de la transmission du cas Menuibois}

\begin{tabular}{|c|c|c|c|c|}
\hline Dates & Phase & Déroulement de la phase & $\begin{array}{c}\text { Rôle du } \\
\text { prédécesseur }\end{array}$ & $\begin{array}{c}\text { Rôle des } \\
\text { successeurs }\end{array}$ \\
\hline Mars 2002 & $\begin{array}{c}\text { Début officiel } \\
\text { du processus }\end{array}$ & $\begin{array}{l}\text { A l'approche de l'âge de la retraite, G.B. propose à ses } \\
\text { deux fils de reprendre l'entreprise familiale. Bien que } \\
\text { cette date marque le début «visible» du processus, G.B. } \\
\text { déclare avoir sans cesse pensé à sa succession. Une } \\
\text { contrainte physique (allergie à la poussière) empêche } \\
\text { son fils aîné de reprendre l'entreprise mais le cadet, } \\
\text { R.B., accepte de réfléchir à la proposition }\end{array}$ & Initiateur & - \\
\hline $\begin{array}{c}\text { Septembre } \\
2002\end{array}$ & \multirow{3}{*}{$\begin{array}{l}\text { Intégration } \\
\text { du successeur } \\
\text { familiale dans } \\
\text { l'entreprise } \\
\text { et constitution } \\
\text { de l'équipe } \\
\text { successorale }\end{array}$} & $\begin{array}{l}\text { Après } 6 \text { mois de réflexion, R.B. accepte la proposition } \\
\text { de son père et rentre dans l'entreprise comme salarié } \\
\text { pour se former au métier de menuisier (via un contrat } \\
\text { emploi-formation). }\end{array}$ & Superviseur & \multirow{3}{*}{$\begin{array}{c}2 \text { Salariés } \\
\text { opérationnels }\end{array}$} \\
\hline Mars 2003 & & $\begin{array}{l}\text { Après un an de réflexion, à laquelle sa femme a participé } \\
\text { ainsi qu'un cabinet juridique, G.B. décide de proposer à } \\
\text { son fils de reprendre l'entreprise avec son unique salarié. } \\
\text { R.B. accepte. }\end{array}$ & Sélectionneur & \\
\hline Juillet 2004 & & R.B. obtient son CAP en candidat libre & Superviseur & \\
\hline $\begin{array}{l}\text { Octobre } \\
2004\end{array}$ & $\begin{array}{l}\text { Début du } \\
\text { règne-conjoint } \\
\text { Début du } \\
\text { transfert de } \\
\text { propriété et de } \\
\text { direction }\end{array}$ & $\begin{array}{l}\text { Formation d'une SARL à } 3 \text { associés (G.B. : } 34 \% \text {, R.B. : } \\
33 \% \text { et } \mathrm{S}: 33 \% \text { ) et } 2 \text { co-gérants (R.B. et S). Les co- } \\
\text { gérants souhaitent que G.B. reste dans l'entreprise pour } \\
\text { une phase de transition et qu'il cède ses parts petit à petit }\end{array}$ & $\begin{array}{l}\text { Conçoit le } \\
\text { montage juri- } \\
\text { dico-financier }\end{array}$ & \multirow{3}{*}{2 Co-gérants } \\
\hline $\begin{array}{c}\text { Novembre } \\
2004\end{array}$ & $\begin{array}{l}\text { Déménage- } \\
\text { ment de } \\
\text { l'entreprise } \\
\text { pour } \\
\text { officialiser la } \\
\text { transmission }\end{array}$ & $\begin{array}{l}\text { Afin de matérialiser le changement de propriétaire aux } \\
\text { yeux des clients et de pouvoir réorganiser les locaux } \\
\text { comme ils le souhaitent, les nouveaux propriétaires } \\
\text { décident de changer la localisation géographique de } \\
\text { l'entreprise. La famille B. achète un local (en SCI) et } \\
\text { le loue à la SARL. Une fête est organisée dans ces nou- } \\
\text { veaux locaux pour marquer les } 25 \text { ans de l'entreprise et } \\
\text { la transmission. }\end{array}$ & $\begin{array}{c}\text { Facilitateur } \\
\text { avec les } \\
\text { banques }\end{array}$ & \\
\hline $\begin{array}{c}\text { Décembre } \\
2004-\text { mai } \\
2005\end{array}$ & $\begin{array}{l}\text { Mise en place } \\
\text { des } \\
\text { changements } \\
\text { post-reprise }\end{array}$ & $\begin{array}{l}\text { Les repreneurs achètent de nouvelles machines et réor- } \\
\text { ganisent la société. R.B.. met à contribution ce qu'on lui } \\
\text { a enseigné dans le cadre de ses études universitaires et } \\
\text { apporte de nombreuses modifications dans le fonctionne- } \\
\text { ment de l'entreprise, à la fois dans l'atelier (il améliore } \\
\text { les méthodes de fabrication), mais aussi en informatisant } \\
\text { les procédures de gestion. Il développe de nouveaux } \\
\text { services à la clientèle. Partage des clients entre R.B. et } \\
\text { S. Le cédant G.B. se déplace en clientèle tour à tour avec } \\
\text { chacun de ses successeurs et leur transmet petit à petit } \\
\text { son savoir-faire en fabrication. }\end{array}$ & $\begin{array}{c}\text { Professeur } \\
\text { Collaborateur } \\
\text { Intermédiaire } \\
\text { avec les clients } \\
\text { et fournisseurs } \\
\text { clés }\end{array}$ & \\
\hline
\end{tabular}




\begin{tabular}{|c|c|c|c|c|}
\hline Juin 2005 & \multirow{5}{*}{$\begin{array}{l}\text { Tensions dans } \\
\text { l'équipe } \\
\text { successorale }\end{array}$} & $\begin{array}{l}\text { Les premières tensions apparaissent entre } \\
\text { les deux dirigeants. }\end{array}$ & & \multirow{5}{*}{2 Co-gérants } \\
\hline $\begin{array}{l}\text { Juillet } 2005 \\
\quad-\text { avril } \\
2006\end{array}$ & & $\begin{array}{l}\text { L'entente entre les deux successeurs se dégrade progres- } \\
\text { sivement et on assiste à une division en deux camps : } \\
\text { d'un côté le cédant et son fils, de l'autre l'ancien salarié } \\
\text { S. à qui l'on reproche son manque de rigueur, son faible } \\
\text { investissement dans le travail et son problème de } \\
\text { communication interne et externe (avec la clientèle). }\end{array}$ & & \\
\hline Mai 2006 & & $\begin{array}{l}\text { Embauche d'un salarié (50 ans) qui a du mal à suivre } \\
\text { l'organisation en place (une entreprise à } 3 \text { têtes) et à se } \\
\text { faire diriger par des dirigeants plus jeunes que lui. }\end{array}$ & & \\
\hline Aout 2006 & & $\begin{array}{l}\text { G.B. organise une réunion de crise avec ses } 2 \text { associés } \\
\text { pour mettre à plat les problèmes. Accalmie temporaire. }\end{array}$ & \multirow[b]{2}{*}{ Médiateur } & \\
\hline $\begin{array}{l}\text { Septembre } \\
\text { à novembre } \\
2006\end{array}$ & & $\begin{array}{l}\text { Les tensions semblent s'apaiser mais les problèmes de } \\
\text { fond demeurent : les deux successeurs ne parviennent } \\
\text { pas à travailler ensemble. }\end{array}$ & & \\
\hline $\begin{array}{l}\text { Décembre } \\
2006\end{array}$ & $\begin{array}{l}\text { Dissolution } \\
\text { de l'équipe } \\
\text { successorale }\end{array}$ & $\begin{array}{l}\text { S. démissionne du poste de co-gérant et cède ses parts } \\
\text { à R.B., après une âpre discussion sur leur valeur. R.B. } \\
\text { doit également racheter la moitié du matériel puisque les } \\
\text { achats avaient été effectués sur les } 2 \text { têtes. S. reprend son } \\
\text { statut de salarié }\end{array}$ & \multirow{2}{*}{$\begin{array}{l}\text { Support } \\
\text { technique } \\
\text { et conseil }\end{array}$} & $\begin{array}{l}\text { Un gérant }+ \\
\text { Un salarié }\end{array}$ \\
\hline $\begin{array}{l}\text { Décembre } \\
2007\end{array}$ & Départ de S. & $\begin{array}{l}\text { S. ne parvenant pas à s'accommoder du statut de salarié } \\
\text { après avoir occupé la fonction de dirigeant, il quitte défi- } \\
\text { nitivement l'entreprise. G.B. continue à se rendre tous } \\
\text { les jours dans l'entreprise }\end{array}$ & & Un gérant \\
\hline
\end{tabular}

fait en revanche apparaître d'autres types de motivations, liées notamment à la taille plus importante de la structure transmise qui entraîne des problématiques de financement et de risques différents. La constitution d'une équipe permet de diviser le coût d'acquisition de l'entreprise et ainsi de partager des risques financiers plus importants. D'un point de vue plus psychologique, elle réduit les risques perçus de la succession et facilite le "passage à l'acte», notamment pour les salariés qui doivent changer de statut et se mettre à diriger leurs anciens collègues. Il est également plus aisé pour eux de se lancer dans cette aventure collective avec des collègues avec lesquels ils entretiennent une relation d'amitié de longue date.

Néanmoins, ce qu'il est important de noter dans ces deux cas, c'est l'influence déterminante du cédant. En effet, c'est non seulement lui qui est véritablement à l'origine $\mathrm{du}$ fait de vouloir transmettre l'entreprise familiale plutôt à une équipe qu'à un seul individu, mais c'est également lui qui désigne les membres de cette équipe. Ainsi, dans les deux cas étudiés, même si chronologiquement l'entreprise n'a pas toujours été proposée en priorité à un enfant, ce dernier a néanmoins été très rapidement rajouté dans l'équipe de successeurs. C.M. (cédant de Mécanix) explique ainsi ses motivations : «j'ai d'abord proposé l'entreprise à mes trois salariés. C'est mon patron qui m'avait aidé à créer mon entreprise quand j'étais jeune. Il fallait donc que je donne aussi la possibilité à mes salariés de devenir chefs d'entreprise. Mais c'est aussi normal de mettre ma fille dans l'équipe pour lui donner sa chance, comme je le fais pour les autres salariés. Ils n'avaient de toute façon personne à proposer pour gérer l'entreprise, ils pensaient certainement pouvoir le faire seuls. Mais je pense que G. leur apportera des compétences qu'ils n'ont pas». Ensuite, le choix de ne pas transmettre seulement à un enfant mais à une équipe composée également de salariés de l'entreprise peut être motivé par d'autres raisons que la nécessité de «leur mettre un pied à l'étrier pour qu'ils deviennent des chefs d'entreprise»(C.M., Mécanix). Au niveau affectif, si les cédants veulent assurer la continuité de l'affaire familiale, ils sont néanmoins conscients de la difficulté du «métier» de dirigeant-propriétaire de PME. Ils reconnaissent également que les «temps ont changé» et que les nouvelles générations ne sont pas toujours aussi enclines à privilégier leur vie professionnelle au détriment de leur vie privée. Souhaitant préserver leurs enfants de ces difficultés, ils estiment qu'une transmission à une équipe de successeurs est préférable. Selon eux, ce partage du travail à accomplir devrait permettre à chaque successeur de conserver un équilibre vie professionnelle - vie familiale. Comme l'explique G.B., le cédant de Menuibois : «quand mon fils a finalement accepté ma proposition de reprendre l'entreprise, je n'en ai pas dormi de la nuit. J'ai repensé à l'investissement que représentait le fait d'être un patron, puis j'ai pensé à $R$. et à sa femme qui venaient d'avoir un bébé; ça m'a fait paniquer. On en a alors longuement parlé avec ma femme et on a pensé que s'ils reprenaient à deux, 


\section{TABLEAU 4}

\section{Présentation des profils et motivations des acteurs en début de processus}

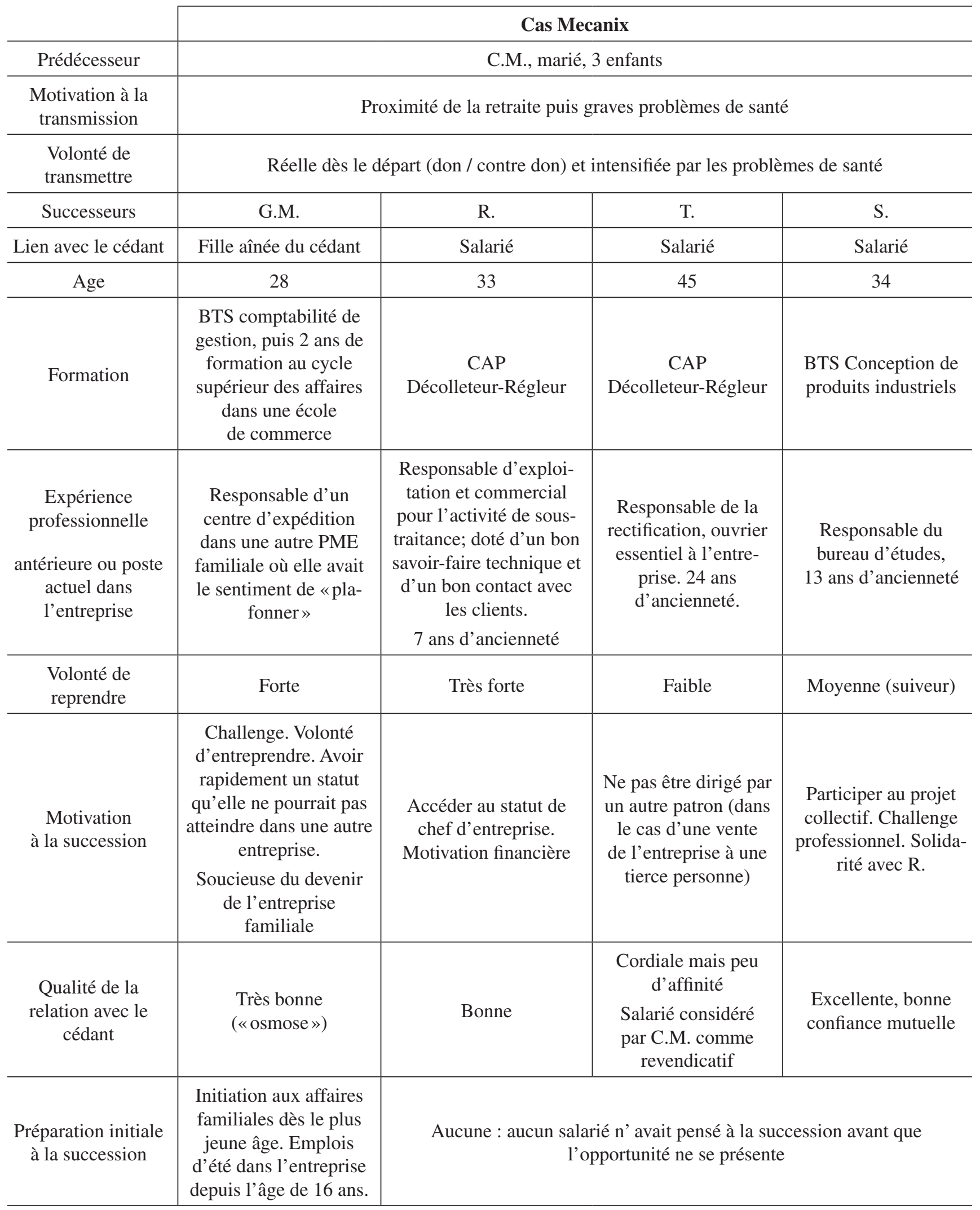




\begin{tabular}{|c|c|c|}
\hline & \multicolumn{2}{|c|}{ Cas Menuibois } \\
\hline Prédécesseur & \multicolumn{2}{|c|}{ GB, marié, 2 enfants } \\
\hline $\begin{array}{l}\text { Motivation à la trans- } \\
\text { mission }\end{array}$ & \multicolumn{2}{|r|}{ Retraite } \\
\hline Volonté de transmettre & \multicolumn{2}{|c|}{$\begin{array}{l}\text { Forte car départ à la retraite proche; volonté de transmettre une œuvre qu'il a construite de } \\
\text { toute pièce et de réaliser son patrimoine }\end{array}$} \\
\hline Successeurs & R.B. & S. \\
\hline Lien avec le cédant & Fils cadet du cédant & Salarié \\
\hline Age & 23 & 23 \\
\hline Formation & DUT Génie Mécanique & CAP Menuiserie et Bac pro Technicien menuisier \\
\hline $\begin{array}{l}\text { Expérience profession- } \\
\text { nelle } \\
\text { antérieure ou poste } \\
\text { actuel dans l'entreprise }\end{array}$ & $\begin{array}{l}2 \text { ans dans un bureau d'études } \\
\text { de Mécanique Générale }\end{array}$ & 10 ans d'ancienneté dans la menuiserie \\
\hline Volonté de reprendre & Forte & Moyenne \\
\hline $\begin{array}{l}\text { Motivation à la suc- } \\
\text { cession }\end{array}$ & $\begin{array}{l}\text { Challenge, défi. Diriger une entreprise où } \\
\text { l'on fait tout de } \mathrm{A} \text { à } \mathrm{Z}\end{array}$ & $\begin{array}{l}\text { Devenir son propre patron (avoir son affaire). } \\
\text { Motivation financière (gagner plus d'argent) }\end{array}$ \\
\hline $\begin{array}{l}\text { Qualité de la relation } \\
\text { avec le cédant }\end{array}$ & Très Bonne & Assez Bonne \\
\hline $\begin{array}{l}\text { Préparation initiale à la } \\
\text { succession }\end{array}$ & $\begin{array}{l}\text { A toujours «donné un coup de main » à } \\
\text { son père pendant les vacances }\end{array}$ & $\begin{array}{l}\text { Aucune, il n'avait pas songé à reprendre mais } \\
\text { plutôt à créer sa propre affaire }\end{array}$ \\
\hline
\end{tabular}

ce serait peut-être plus facile pour eux de concilier leur vie de chef d'entreprise avec leurs obligations familiales. Ils pourraient se partager le travail».

Pour ce qui est du choix des salariés composant cette équipe de successeurs, le cédant de Mécanix a eu l'occasion de les tester pendant un temps relativement long avant la mise en œuvre de la succession. Comme il l'exprime, «pour pouvoir commencer à créer cette dynamique (la succession), il vaut mieux que le patron se soit intéressé à ses salariés et ait instauré une certaine relation plutôt que de découvrir au dernier moment qu'il y a des hommes derrière les machines. Moi je me suis toujours intéressé à mes salariés, je les connais bien, et ce n'était pas difficile d'identifier des successeurs potentiels. Après avoir proposé l'affaire aux deux plus anciens, C. et G., qui n'ont pas voulu tenter l'aventure, je savais que les seuls autres salariés à pouvoir y aller étaient $R$., T. et S. Ils travaillaient dans l'entreprise depuis longtemps et j'avais eu le temps de bien les observer. Et puis, ils s'entendaient bien ensemble». Dans le cas Menuibois, si idéalement le cédant aurait préféré transmettre l'entreprise à ses deux enfants, on trouve une autre motivation à l'inclusion d'un salarié dans l'équipe que le seul fait que celui-ci soit considéré comme «une solution de rechange» : la réduction du risque lié à la présence d'un unique salarié dans la structure. En effet, cet employé aurait pu quitter l'entreprise suite au changement de direction, notamment pour créer une structure concurrente, ce qui aurait nuit à la pérennité de la TPE familiale. La constitution d'une équipe composée de successeurs familiaux et de salariés apparaît donc au final, pour les cédants, non seulement comme une façon de protéger les membres de la famille mais aussi comme un gage de réussite de la succession et de pérennité de l'entreprise transmise. Notons pour terminer que dans les deux cas les femmes des cédants ont contribué à la prise de décision concernant le choix des successeurs. Dans le cas Mécanix, c'est U.M. qui a décidé de laisser sa place d'assistante de direction à sa fille et dans le cas Menuibois c'est notamment la femme de G.B. qui, au-delà de ses responsabilités de gestion comptable de l'entreprise, avait à cour que son fils puisse concilier vie professionnelle et vie familiale.

\section{LA COMPOSITION DES ÉQUIPES SUCCESSORALES CRÉATRICE DE CONFLITS COGNITIFS ET AFFECTIFS}

Etant donné le mode de sélection des membres de l'équipe (membres choisis par le cédant), on ne peut parler de liens forts unissant les individus dans aucune des deux équipes. Dans le cas Menuibois, les deux successeurs ont le même âge et se connaissent : le fils du cédant a eu l'occasion de rencontrer plusieurs fois le salarié lorsqu'il est venu travailler dans l'entreprise pendant les vacances scolaires. Il a même été dans sa classe au collège. Cependant aucun lien d'amitié ne les unit. Chez Mecanix, un tel lien existe entre les 3 salariés de la structure, du fait de longues années de travail en commun. Comme le déclare le successeur S. 
(Mécanix) : «avec $R$, ça fait 7 ans qu'on bosse ensemble et avec T, 15 ans! On se connait bien, on connait nos défauts et nos qualités. On sait ce qui risque de nous mettre en rogne et en général on évite d'aborder ces sujets-là. (...) Tout seul, je n'y serai jamais allé, mais avec eux deux, et avec $C$ et $G$ (deux autres salariés plus anciens qui ont refusé la reprise), on est quand même les piliers de l'entreprise». En revanche, aucun lien n'unit ces trois salariés à la fille du cédant (G.M.) qui leur parait différente à plusieurs titres : c'est une femme, dans un monde plutôt masculin (secteur de la mécanique), qui a fait des études supérieures, dans le domaine de la gestion (et non dans celui de la technique). Le successeur-salarié R. s’exprime ainsi à son sujet : « $G M$, c'est un peu une fille à papa. Son père lui a payé une école privée pour faire ses études et elle croit qu'elle peut directement appliquer ses principes appris à l'école pour diriger une entreprise alors qu'elle ne connait rien au métier.»

En termes de complémentarité des ressources et compétences, les deux équipes diffèrent. Les successeurs de Mécanix paraissent à première vue complémentaires, car ils réunissent différentes fonctions de l'entreprise (gestion administrative et financière pour la fille du cédant et partie plus technique pour les autres successeurs). Présenté comme une force en termes d'accès à des compétences variées, cette disparité entraîne néanmoins au quotidien des conflits importants sur les orientations stratégiques à donner. Dans le cas Menuibois, on relève plutôt une certaine redondance des compétences. Ainsi les deux successeurs étant titulaires d'un CAP de Menuiserie, ils se répartissent les clients plutôt que les fonctions. Toutefois, la différence de niveaux d'étude provoque des incompréhensions entre les deux successeurs. R.B. (fils du cédant) : «En termes d'organisation, on se partage les clients pour pouvoir travailler de A à Z pour un client et ne pas toujours faire la même chose. Mais c'est vrai que c'est plutôt moi qui essaye d'apporter des améliorations au fonctionnement. Par exemple, j'ai créé des fichiers Excel et Word pour archiver l'information (des courriers types, des fichiers de fabrication pour chiffrer ce qui revient régulièrement, des prévisionnels...). J'ai créé un planning visuel qui est affiché sur les murs du bureau pour toujours savoir qui fait quoi et pour organiser le travail. J'ai développé la proposition de dessins et de plans avant les travaux pour les clients. S.(l'ancien salarié), lui, ne voit pas à quoi tout ça sert. Il a eu l'habitude de bosser avec mon père qui ne sait pas se servir d'un ordinateur alors il ne voit pas pourquoi il faut que les choses changent. Même en fabrication, alors qu'il a plus d'expérience que moi, c'est moi qui améliore les méthodes grâce à ce que j'ai appris en DUT Génie Mécanique. En métallurgie, on est plus exigeant qu'en menuiserie, ça exige plus de précision. Du coup, on a fait de gros progrès en qualité.»

Les deux équipes successorales étudiées étaient également porteuses de différences en matière d'expérience

7. Les conflits cognitifs sont orientés sur les tâches et sur la façon d'atteindre les objectifs, tandis que les conflits affectifs portent davantage sectorielle : les enfants des cédants avaient dans les deux cas moins d'expérience du secteur d'activité de l'entreprise reprise que les autres membres salariés de l'équipe de succession. Ce manque d'expertise s'est avéré être une source de conflit supplémentaire, le successeur familial manquant, dans le cas Mecanix, de légitimité aux yeux des autres membres de l'équipe successorale. Le successeur R. (salarié de Mecanix) décrit ainsi G.M., la fille du cédant : «elle est arrivée dans l'entreprise avec ses certitudes sur le management, ses recettes de cuisine toutes prêtes, sans s'intéresser aux hommes, à leur histoire et leur expérience dans l'entreprise». Cette dernière, de son côté, justifie son manque d'expérience différemment : «mon père a mal habitué les salariés. Il a toujours adopté un comportement plus proche de celui d'un salarié, présent tous les jours dans l'entreprise, portant la blouse et participant à la production, que de celui d'un dirigeant qui prend du recul, gère l'entreprise et coordonne l'activité. Plus proche du technicien et de l'ouvrier qu'il était par le passé que du patron, il se devait toujours de donner l'exemple. Résultat, R. et T. (deux autres successeurs salariés) n'acceptent pas que quelqu'un comme moi, qui ne maitrise pas la technique, ait des idées de développement et les dirige». Les points de dissension sont quelque peu différents dans le cas Menuibois, mais dénotent également de différences de conception quant à la direction de l'entreprise. Voici comment le fils R.B. s'exprime au sujet de l'autre successeur-salarié : «tant qu'il est encadré, ça va, mais lorsqu'il faut trouver des solutions et réaliser un travail de A à $Z$, il «bricole», et tout est à refaire. (...) S. est trop individualiste, il vit de son côté, il veut choisir ses clients, et on a l'impression que deux entreprises vivent sous le même toit». L'autre successeur S. affiche également son mécontentement face à la situation : «on passe notre temps à discuter, à se mettre au point. A trois personnes, c'est trop compliqué, surtout que même s'il ne veut pas l'avouer, G.B. (le cédant) a tendance à toujours être d'accord avec son fils plutôt qu'avec moi». Les deux équipes ont donc été confrontées à plusieurs conflits cognitifs et affectifs ${ }^{7}$ qui n'ont pas pu être dépassés du fait de l'absence de liens forts unissant les membres de l'équipe. Ces conflits ont été nuisibles au bon fonctionnement de ces équipes successorales. Dans le cas Mecanix, seule la solidarité entre les trois successeurs anciens salariés est demeurée quasi-intacte. Comme le souligne le successeur-salarié T. : «moi au départ, je n'étais pas super motivé pour reprendre l'entreprise. C'est beaucoup de soucis d'être patron. J'ai accepté pour donner un coup de main à $R$. et $S$. qui avaient envie d'essayer. Quand je vois comme c'est compliqué aujourd'hui, en particulier avec G.M (fille du cédant), je me dis que je n'avais pas tort. Mais bon, je me suis engagé, je ne peux pas les laisser tomber».

sur des incompatibilités de personnalités (Francis et Sandberg, 2000). 
DES INÉGALITÉS DES MEMBRES DE L'ÉQUIPE QUI ENGENDRENT DES DIFFICULTÉS DE FONCTIONNEMENT

Dans les deux cas étudiés, la répartition du capital s'est voulue égalitaire au départ. Il y avait là une volonté affichée des cédants à la fois de montrer leur neutralité vis-à-vis de chacun des membres de l'équipe et de faciliter le financement de la succession. Ce partage égalitaire du capital a été de pair avec le partage du travail qui s'est lui aussi voulu objectif dans un premier temps. Ainsi chez Mécanix, chaque successeur est demeuré dans son domaine de compétence (commercial, gestion ou technique). Au sein de l'entreprise Menuibois, les deux successeurs se sont également partagé les chantiers, au fur et à mesure que l'entreprise décrochait de nouveaux clients. Toutefois ce fonctionnement démocratique n'a pas donné de résultats optimaux. Il s'est traduit par la difficulté à faire émerger un leader chez Mecanix (la fille du cédant ayant officiellement été nommée au poste de dirigeante) et par l'imposition finale des choix du fils du cédant chez Mécabois. Cette égalité apparente sur le papier -le contrat- masque en outre une grande inégalité des membres en termes de lien de parenté avec le cédant. Ainsi même si pour les cédants, les différents types de successeurs étaient jugés égaux, les enfants ont eu tendance à être avantagés, au moins de façon implicite en ayant un accès privilégié à certaines informations. En outre, même après avoir quitté officiellement leur poste de dirigeant, les cédants ne pouvaient s'empêcher de rester présents, soit physiquement dans l'entreprise (Menuibois), soit en arrière-plan, en tirant les ficelles en coulisses (Mécanix). Or cette préférence constatée pour la famille ${ }^{8}$ a déclenché un cercle vicieux de défiance au sein de l'équipe de successeurs, opposant les membres de la famille à ceux n'en faisant pas partie. Il n'est ainsi pas étonnant de constater que les deux successeurs qui ont tenté de s'imposer comme leaders soient les enfants des cédants, même si on peut considérer qu'il s'agissait également des successeurs les plus motivés par l'opération. En effet, dans la succession Mécanix, la fille du cédant a d'abord été désignée (plus ou moins par son père) pour occuper le poste officiel de dirigeante de l'entreprise. Mais ce leadership n'a pas été reconnu par les autres membres de l'équipe successorale, les membres salariés exerçant une certaine résistance quant aux choix stratégiques décidés par cette dernière. Chez Menuibois, cet entrepreneur leader a également été le fils du cédant. Cette prise de leadership s'est avérée apparemment plus facile car l'équipe successorale n'était composée que d'un seul autre membre. Toutefois, elle n'a pas forcément été bien vécue par le successeur salarié qui n'adhérait pas, entre autres, aux nouvelles méthodes de travail mises en place. On n'est donc pas, dans les deux cas, face à des leaders naturels reconnus au sein de l'équipe. On peut là encore estimer que c'est le processus de constitution des équipes successorales qui

8. Notons toutefois que cette préférence repose également sur un certain nombre de faits pouvant s'avérer plus «objectifs». En effet, dans les deux cas étudiés, ce sont les enfants du cédant qui se sont montrés les plus motivés par la reprise de l'entreprise familiale et qui ont été les plus actifs est à l'origine de ce dysfonctionnement. Une équipe qui se serait constituée d'elle-même, sur la base d'un vrai projet de succession, aurait sans doute eu moins de mal à désigner un leader. Ce leader a d'ailleurs pu émerger naturellement dans le cas Mécanix, après le départ de la fille du cédant, le salarié R. ayant plus de légitimité aux yeux de ses autres collègues successeurs pour assumer le poste de dirigeant.

Le tableau $\mathrm{n}^{\circ} 5$ résume les résultats de l'analyse de ces deux cas sur les différentes dimensions composant notre cadre d'analyse.

\section{Formation de l'équipe de successeurs et difficultés de la succession : quels enseignements?}

Les travaux empiriques sur les équipes successorales étant à ce jour encore peu nombreux, les deux études de cas réalisées nous ont permis de mieux comprendre comment leur mode de formation pouvait être porteur de difficultés. On peut ainsi retirer trois principaux enseignements de cette recherche.

\section{MODE DE CONSTITUTION ET SUCCÈS DE L'ÉQUIPE SUCCESSORALE}

En matière de mode de constitution de l'équipe successorale, les deux cas étudiés ne s'apparentent à aucune des situations identifiées par Kamm et Nurick (1993) dans le cadre de la création ex nihilo. En effet, ce n'est ni un successeur qui a un projet de reprise d'entreprise et qui cherche ensuite des partenaires («lead entrepreneur approach»), ni une équipe qui préexiste et qui recherche une opportunité de reprise («group approach»). Se manifeste ici sans doute l'une des spécificités de la succession par rapport à la création, c'est la prégnance forte du cédant sur le déroulement du processus, influence centrale déjà largement évoquée dans la littérature sur la transmission d'entreprise (Mouline, 2000; Cadieux, 2007). Si les chercheurs s'accordent à reconnaitre que le cédant est généralement la personne qui initie le plus souvent le processus de succession (Handler, 1994; Lambrecht, 2005; Cadieux, 2007), nous pouvons ici constater que dans le cas de la transmission à une équipe, il peut également adopter un nouveau rôle : celui de sélectionneur des membres de cette équipe. Le cédant va sélectionner les membres qui lui paraissent le plus pertinent par rapport à son projet de transmission, ceux dont il se sent le plus proche et avec lesquels il entretient une relation de confiance. La littérature sur les successions familiales soulève fréquemment la nécessaire bonne entente et le respect mutuel devant exister entre un cédant et son successeur (Cater et Justis, 2009). Cependant, dans le cas de la

dans le processus de succession, notamment en voulant la développer ou en mettant en place (ou tenté de mettre en place dans le cas Mécanix) de nombreux changements opérationnels ou stratégiques. 


\section{TABLEAU 5}

\section{Analyse de la constitution des deux équipes de successeurs}

\begin{tabular}{|c|c|c|}
\hline & Mécanix & Menuibois \\
\hline $\begin{array}{l}\text { Complémentarité des } \\
\text { membres de l'équipe } \\
\text { en termes de } \\
\text { compétences }\end{array}$ & $\begin{array}{c}\text { Forte : gestion, production, } \\
\text { bureau d'étude, commercial } \\
\text { Les successeurs se répartissent le travail, } \\
\text { chacun restant dans son domaine } \\
\text { de prédilection }\end{array}$ & $\begin{array}{l}\text { Redondance sur la partie production dès lors que } \\
\text { R.B. a obtenu son CAP de menuiserie. Les deux } \\
\text { successeurs réalisent des tâches similaires dans } \\
\text { l'entreprise et se partagent les chantiers }\end{array}$ \\
\hline $\begin{array}{l}\text { Nature des liens } \\
\text { entre les membres } \\
\text { de l'équipe }\end{array}$ & \begin{tabular}{|l} 
Faible entre la fille du cédant (G.M.) \\
et les 3 autres membres de l'équipe. \\
Forte entre les 3 successeurs salariés (amitié \\
et expérience professionnelle partagée)
\end{tabular} & $\begin{array}{l}\text { Faible (les deux successeurs avaient seulement } \\
\text { été dans la même classe au collège) }\end{array}$ \\
\hline $\begin{array}{l}\text { Modalité de } \\
\text { constitution de } \\
\text { l'équipe }\end{array}$ & $\begin{array}{l}\text { Le projet de succession déclenche } \\
\text { la constitution de l'équipe. } \\
\text { Le cédant sélectionne les membres } \\
\text { de l'équipe }\end{array}$ & $\begin{array}{l}\text { Le projet de succession déclenche la constitution } \\
\text { de l'équipe } \\
\text { Le cédant sélectionne les membres de l'équipe }\end{array}$ \\
\hline $\begin{array}{l}\text { Motivations des } \\
\text { successeurs à former } \\
\text { une équipe }\end{array}$ & $\begin{array}{c}\text { - Financières : partager le coût et les risques } \\
\text { d'acquisition de la structure } \\
\text { - Partager la masse de travail } \\
\text { - Compenser le manque de motivation } \\
\text { individuelle par la solidarité des membres } \\
\text { et diminuer l'anxiété liée au changement } \\
\text { de statut (pour les salariés) }\end{array}$ & - Meilleure répartition du travail \\
\hline $\begin{array}{l}\text { Motivations du } \\
\text { cédant à transmettre } \\
\text { à une équipe de } \\
\text { successeurs }\end{array}$ & $\begin{array}{l}\text { - Raisons affectives : donner sa chance } \\
\text { à tous (enfants comme salariés) } \\
\text { - Complémentarité perçue des compétences }\end{array}$ & $\begin{array}{l}\text { - Faciliter la transmission à son fils (diminuer } \\
\text { la masse de travail et lui permettre de mieux } \\
\text { concilier vies personnelle et professionnelle) } \\
\text { - Empêcher son salarié de monter une structure } \\
\text { concurrente }\end{array}$ \\
\hline $\begin{array}{l}\text { Difficultés } \\
\text { rencontrées par } \\
\text { l’équipe }\end{array}$ & $\begin{array}{l}\text { - Conflits sur les orientations à donner } \\
\text { à l'entreprise et sur le leadership } \\
\text { - Problèmes de coordination } \\
\text { - Absence de confiance } \\
\rightarrow \text { Conflits cognitifs et affectifs qui } \\
\text { aboutissent au départ d'un des membres } \\
\text { de l'équipe }\end{array}$ & $\begin{array}{c}\text { - Conflits sur les orientations à donner à l'entre- } \\
\text { prise, les méthodes de travail et les changements } \\
\text { à opérer dans la structure } \\
\text { - Problèmes de coordination } \\
\text { - Manque de performance perçu d'un des } \\
\text { membres de l'équipe (salarié) au regard des } \\
\text { attentes de l'autre successeur (fils) } \\
\rightarrow \text { Conflits cognitifs et affectifs }\end{array}$ \\
\hline $\begin{array}{l}\text { Facteurs d'échec } \\
\text { du fonctionnement } \\
\text { des équipes }\end{array}$ & $\begin{array}{l}\text { - Trop grande hétérogénéité des membres } \\
\text { aux niveaux fonctionnel, de l'expérience } \\
\text { sectorielle et du genre, mais surtout au } \\
\text { niveau du lien de parenté avec le cédant } \\
\text { - Absence de liens forts et donc de solidarité } \\
\text { entre les membres (présence de deux clans; } \\
\text { incompatibilité de personnalités) liée au } \\
\text { mode de constitution de l'équipe } \\
\rightarrow \text { Objectifs et valeurs incompatibles } \\
\text { Manque de confiance entre les «deux clans» }\end{array}$ & $\begin{array}{c}\text { - Trop grande hétérogénéité des membres } \\
\text { au niveau du lien de parenté avec le cédant } \\
\rightarrow \text { Cédant ayant tendance à privilégier } \\
\text { implicitement son fils } \\
\text { - Absence de liens forts et donc de solidarité } \\
\text { entre les membres de l'équipe }\end{array}$ \\
\hline
\end{tabular}


transmission à une équipe, la relation cédant-successeur n'est pas suffisante. Il faut que les membres de l'équipe entretiennent également des rapports de qualité pour dépasser les conflits inhérents au partage du pouvoir. Or cela est plus difficile lorsque les membres ne se choisissent pas eux-mêmes. Il nous semble alors préférable que le choix de reprendre en équipe soit un choix délibéré et clair pour tous les membres de l'équipe (et non pour le seul cédant). Ces derniers doivent être animés d'un réel sentiment d'appartenance à l'équipe (Ensley et Pearson, 2005; Harper, 2008) et non se référer sans cesse à un objectif individuel ou au projet du cédant. Leurs motivations doivent être compatibles (Kamm et al, 1990) et ils doivent avoir conscience que la combinaison de leurs actions peut les amener à être plus efficace dans le déroulement de la succession. Les équipes successorales étudiées auraient sans doute été plus performantes si elles s'étaient constituées d'elles-mêmes, sur la base d'un leader qui convainc les autres membres de le rejoindre. Ici c'est le cédant qui a joué le rôle de leader dans la constitution des équipes. Or comme l'issue de la succession est que cet individu quitte l'entreprise, ce rôle de leader n'est pas forcément adapté. Ceci nous amène à formuler notre première proposition :

Proposition 1 : pour le succès de la transmission, le cédant doit laisser l'équipe successorale se former d'elle-même.

\section{HÉTÉROGÉNÉITÉ DE L'ÉQUIPE ET DIFFICULTÉS}

Une autre difficulté constatée dans cette étude a été liée à une trop grande hétérogénéité des membres des équipes. Plusieurs auteurs ayant travaillé sur les équipes entrepreneuriales (Eisenhardt et Schoonhoven, 1990; Ucsabaran et al , 2003; Moreau, 2006) ont souligné les avantages (diversité de connaissances et de savoir-faire, plus grande capacité d'innovation...) et les limites de cette hétérogénéité (division du groupe, complication des liens, conflits destructeurs...). Kamm et al (1990), par exemple, plaident pour un bon équilibre en termes d'expertise fonctionnelle, de compétences de management et d'expérience au sein d'une équipe d'entrepreneurs. Dans les deux cas étudiés, on constate que le cédant, qui a été à l'origine de la constitution des équipes, a peut-être trop été à la recherche d'un « mouton à cinq pattes », en essayant de mêler à la fois rationalité économique (réunir des individus aux compétences variées et conserver au sein de la structure les salariés clés de l'entreprise) et affect (primauté aux liens de parenté et altruisme envers la famille; Royer et al, 2005; Cruz et al, 2013; qui se superposent parfois à une motivation psychologique de don/contre-don envers les salariés; Lobet, 2006). Dans les entreprises familiales, il est courant que les obligations familiales interfèrent avec la rationalité économique (Whyte, 1996; Westhead, 1997; Mouline, 2000; Stewart, 2003; Cruz et al, 2013). Il semble cependant illusoire de vouloir satisfaire l'ensemble des parties prenantes en les incluant dans une seule et même équipe de successeurs.
Dans les cas étudiés, cela a abouti à la réunion de membres aux statuts trop différents. Le fait que ces membres ne soient pas sur le même plan, du point de vue de la relation avec le cédant, a introduit une certaine inégalité entre eux, même si sur d'autres aspects les successeurs pouvaient être considérés comme égaux (par exemple en matière de partage du capital). Les cédants n'ont pu s'empêcher d'avoir un rôle ambigu et au final de privilégier leurs enfants, au moins de façon implicite ou en tout cas perçu comme tel par les salariés. L'hétérogénéité en termes de liens avec le cédant est donc porteuse de difficultés importantes, en particulier car elle crée des problèmes de défiance entre les individus. Elle introduit des failles («faultlines ») créant des divisions au sein des équipes (Schjoedt et al, 2013). Cette rivalité pouvant exister entre membres et non-membres de la famille a déjà été évoquée dans la littérature sur les PME familiales (De Massis et al, 2008). Cependant elle crée ici de plus grands dysfonctionnements dans la mesure où ces deux types de membres se partagent non seulement la direction mais aussi la propriété de l'entreprise. Ce constat nous amène à formuler notre deuxième proposition :

Proposition 2 : pour le succès des équipes successorales, une homophilie des membres de l'équipe en termes de liens de parenté avec le cédant est préférable.

\section{Proximité Sociale Versus Complémentarité deS MEMBRES DE L'ÉQUIPE}

En ce qui concerne les critères de sélection «objectifs » des membres des équipes, c'est plutôt la complémentarité qui a été mise en avant dans les deux cas, à la fois par les cédants à l'origine de la constitution de ces équipes et par les membres eux-mêmes qui, en conséquence, ne se sont pas vraiment choisis mais ont justifié a posteriori cette composition. Cette complémentarité des savoirs et compétences est souvent perçue par les chercheurs en entrepreneuriat comme un avantage dans certains types d'environnement (Eisenhardt, et al, 1990) ou à certains moments du développement de l'entreprise (Moreau, 2006). Il semblerait qu'en situation de succession, cette complémentarité soit moins porteuse de réussite que la proximité sociale ou des liens forts unissant les membres de l'équipe. Dans le cas Mecanix, la trop grande hétérogénéité évoquée plus haut (en termes d'expériences sectorielles, de formations suivies et de compétences possédées par les membres), bien que séduisante et crédible a priori (Chabaud et Condor, 2009), a engendré de nombreux conflits cognitifs qui se sont superposés à des conflits affectifs. Dans le cas Menuibois, l'expérience de travail en commun ne préexistait pas à la succession et même si les deux successeurs se connaissaient avant la reprise, ce lien ne pouvait pas être qualifié d'amical. L'alchimie n'a pris dans aucune des équipes. Au contraire, l'absence de liens affectifs et de confiance a altéré la communication et le fonctionnement des équipes (Ben-Hafaiedh-Dridi, 2001; Sharma, 2004) et n'a pas pu diminuer les conflits liés aux différences d'objectifs ou de 
valeurs (Francis et Sandberg, 2000). Seuls les trois anciens salariés de Mécanix ont réussi à s'entendre sur la direction à donner à l'entreprise, car ils avaient travaillé côte à côte durant de nombreuses années et avaient ainsi tissé des liens d'amitié. La fréquence de leurs interactions passées leur a permis de développer une vision commune (Francis et Sandberg, 2000; Harper, 2008). Cette amitié a joué le rôle de ciment dans cette partie de l'équipe et a même parfois créé des coûts psychologiques de sortie pour les membres (Francis et Sandberg, 2000; Ucsabaran et al, 2003), notamment pour le successeur le moins motivé au départ (T.). On pourrait alors se demander si la complémentarité des ressources et compétences ne pourrait pas être plus importante en création ex nihilo, où tout est à mettre en place (Harrison et Leitch, 1995; Paturel, 2007; West, 2007; Ucbasaran, 2008) et où l'incertitude est plus élevée (Harper, 2008), que dans le cas d'une succession où l'on bénéficie déjà d'une entreprise en état de fonctionnement. La présence au sein de l'entreprise reprise de salariés aux compétences déjà mobilisables pourrait en effet jouer le rôle de substituts à certaines compétences des successeurs. Toutefois les études récentes portant sur le succès des équipes entrepreneuriales montrent également que la variété des compétences des membres de l'équipe passe au second plan derrière les aspects plus relationnels (Schjoedt et al, 2013). C'est sur la base de ces constats et réflexions que nous formulons notre dernière proposition :

Proposition 3 : Pour le succès d'une transmission à une équipe, il est préférable d'avoir des liens forts entre les membres plutôt qu'une complémentarité des compétences.

\section{Conclusion}

Cette recherche constitue un premier travail empirique sur l'émergence et le fonctionnement des équipes successorales. Les deux études de cas longitudinales réalisées permettent de mettre en lumière plusieurs enseignements sur la façon dont ces équipes se constituent et évoluent dans le temps, sous l'influence des principales parties prenantes. On a ainsi pu constater que, dans le cadre d'une affaire familiale, c'est le cédant qui peut être à l'origine non seulement de la constitution de l'équipe de successeurs mais aussi du choix de ses membres. Ne pouvant faire abstraction de son rôle de «Pater Familias» (Perez-Lizaur, 1997; Mouline, 2000), il peut avoir tendance à vouloir confier l'entreprise à ses enfants, tout en souhaitant les préserver au maximum des difficultés inhérentes au processus de succession et au statut de propriétaire-dirigeant d'une PME. Il va ainsi préférer la transmission à une équipe successorale plutôt qu'à une seule personne, incluant de ce fait dans l'équipe une autre partie prenante clé de la PME familiale : un ou plusieurs salariés. Cependant, malgré une volonté affichée de traiter tous les membres de l'équipe successorale sur un pied d'égalité, le cédant peut, au cours du processus de succession, privilégier plus ou moins implicitement les membres de sa famille au détriment des autres membres de l'équipe successorale. Ce mode de constitution, dans lequel les membres de l'équipe ne se choisissent pas vraiment eux-mêmes, et ce degré d'hétérogénéité trop important des membres de l'équipe en termes de lien de parenté avec le cédant, perturbent le fonctionnement de l'équipe, et donc au final le déroulement de la succession. L'absence de réelle proximité sociale entre les membres ainsi réunis ne permet pas à l'équipe de surmonter les conflits.

L'originalité de cette recherche est par conséquent de mettre en relief le lien entre le mode de constitution d'une équipe successorale et son échec. Ce faisant, elle enrichit deux corpus théoriques. Au niveau de la littérature sur la transmission-reprise, elle apporte des enseignements sur ce mode original de transmission qu'est celle à une équipe de successeurs, jusqu'ici peu étudié. En matière de littérature sur les équipes entrepreneuriales, elle apporte également des connaissances nouvelles sur l'émergence d'un type d'équipe particulier : les équipes successorales. Elle comble ainsi en partie le déficit de littérature portant sur la façon dont ces équipes se forment et évoluent dans le temps (Schjoedt et al, 2013).

En ce qui concerne les implications managériales, ce travail souligne les difficultés liées à une trop grande hétérogénéité des membres de l'équipe successorale. Il invite les cédants attirés par ce mode de succession à réfléchir sur la pertinence de la composition d'une telle équipe et sur leur capacité à faire abstraction des liens qui peuvent les unir à certains successeurs. Le déroulement des deux cas étudiés laissent entrevoir que cette hétérogénéité est un facteur d'échec important et qu'il serait sans doute préférable de ne réunir que des successeurs ayant le même lien de parenté (membres de la famille ou salariés uniquement). Par ailleurs, s'il peut paraître légitime que le cédant choisisse qui va le remplacer à la tête de l'entreprise, il semblerait préférable que l'équipe se constitue d'elle-même sur la base de liens d'amitié et de respect mutuel ou au moins sur une réelle volonté commune de reprendre ensemble l'entreprise.

Malgré ces enseignements, cet article présente un certain nombre de limites dont il faut tenir compte si l'on cherche à généraliser ces résultats. La première est liée au caractère exploratoire de la recherche et au faible nombre de cas étudiés. Les tendances observées méritent d'être validées par un échantillon plus important. Cela ouvre sur une piste de recherche qui mobiliserait une méthodologie quantitative confirmatoire se fondant sur les propositions élaborées ici. En effet, les deux entreprises étudiées ont pour point commun de concerner des activités où le savoir-faire technique prédomine et où les personnes qui le détiennent sont plutôt en situation de force. Le déroulement de la succession aurait peut-être été différent dans des activités tertiaires, où la prégnance du savoir-faire technique est moins importante. Une étude quantitative pourrait ainsi permettre de contrôler l'influence de variables telles que l'appartenance sectorielle de l'entreprise. Ensuite, c'est un cas particulier d'équipes 
successorales qui a été étudié, réunissant à la fois des membres de la famille du cédant et des salariés. Le déroulement de la succession aurait sans doute été différent si ces équipes avaient été plus homogènes en termes de lien de parenté avec le cédant, comme dans le cas d'une reprise par une fratrie ou par des salariés uniquement. De même, dans le cas d'une transmission externe, à des tierces personnes n'ayant aucun lien familial ni d'emploi avec l'entreprise transmise, la constitution des équipes aurait sans doute été très différente et se serait vraisemblablement plus rapprochée de celle des équipes entrepreneuriales plus classiques. D'autres recherches pourraient ainsi être menées sur des équipes successorales plus homogènes (équipes de successeurs composées uniquement de membres de la famille, ou de salariés ou de repreneurs externes). Enfin, deux types de parties prenantes ont été privilégiés dans le recueil des données : les cédants et les successeurs. En effet, si on a pu interviewer un consultant extérieur dans un des cas, nous n'avons pas réalisé d'entretiens avec d'autres salariés que les membres de l'équipe de successeurs, ni avec des parties prenantes extérieures telles que les partenaires commerciaux. L'inclusion de la vision de ces acteurs dans le recueil et l'analyse des données permettrait d'obtenir un regard peut être plus distancié sur le déroulement des successions. En conséquence, cette étude ne constitue qu'un propos d'étape et nécessite des prolongements.

\section{Références}

Aldrich H. E.; Carter, N. M.; Ruef M. (2004). «Teams», in Gartner, W. B., Shaver K.G., Carter N.M., et Reynolds S P.D., Handbook of Entrepreneurial Dynamics. The Process of Business Creation, London : Sage, p. 299-310

Almeida, Heitor; Wolfenzon, Daniel (2006). «A theory of pyramidal ownership and family business groups», Journal of Finance, 61, p. 2637-2680.

Aronoff, C. (1998). «Megatrends in family business», Family Business Review, vol 11, $n^{\circ} 3,181-185$

BARNEY, J. (1991). «Firm resources and sustained competitive advantage », Journal of Management, vol 17 n 1 , p 99-120.

Beckhard, R.; Dyer, W. (1983). «Managing Change in the Family Firm-Issues and Strategies», Sloan Management Review, vol 24, p. 59 - 65

Ben-Hafaiedh-Dridi, C. (2010). «Entrepreneurial team formation : any rationality?», Frontiers of Entrepreneurship Research, vol 30, ${ }^{\circ} 10$, article 1

Bornard, Fabienne; ThÉvenard-Puthod, Catherine (2009). «Mieux comprendre les difficultés d'une reprise externe grâce à l'approche des représentations sociales», Revue Internationale PME, vol 22 n³-4, p.83-108.

Bughin, Christiane; Colot, Olivier; Finet, Alain (2010). «Entreprises familiales et gouvernance cognitive : quelle transmission?», Management \& Avenir, vol 7 n³7, p. 14-33.

BuRT, Ronald S. (1992). Structural holes. The Social Structure of Competition, Cambridge, Harvard University Press.
Cabrera-Suarez, Katiuska (2005) . «Leadership transfer and the successor's development in the family firm,», The Leadership Quarterly, vol 16, p. 71-96

CAdieux, Louise (2007). «Succession in Small and MediumSized Family Businesses : Toward a Typology of Predecessor Roles during and After Instatement of the Successor », Family Business Review, Vol. 20, No. 2, p. 95-109

CAdieux, Louise; Brouard, François (2009). La transmission des $P M E$, Presses de l'Université du Québec, Québec

Cadieux, Louise; Deschamps, Bérangère (2009). «Le repreneuriat, une pratique entrepreneuriale de plus en plus reconnue», Coordination du numéro spécial consacré au repreneuriat, Revue Internationale PME, vol 22, n³ / 4, p.7-12

Cadieux, Louise; Deschamps, Bérangère (2011). Le duo cédantrepreneur, pour une compréhension intégrée du processus de transmission-reprise des PME, PUQ, Québec.

Carlock, R.S.; Ward, J.L. (2001). Strategic Planning for the Family Business : Parallel Planning to Unite the Family and Business. Palgrave Macmillan

Cater, John James; Justis , Robert T. (2009). «The Development of Successors From Followers to Leaders in Small Family Firms : An Exploratory Study», Family Business Review, 22, 109-124.

Cater, John James; Justis Robert, T. (2009). «The Development and implementation of shared leadership in multigenerational family firms », Management Research review, 33, 2, 563-585.

Chabaud, Didier; Condor, Roland (2009). «La formation des équipes entrepreneuriales : une étude exploratoire», Revue Internationale $P M E$, vol $22 \mathrm{n}^{\circ} 1, \mathrm{p} 81-102$.

Chandler, G.N.; Hanks, S.H. (1998). «An examination of the substitutability of founders' human and financial capital in emerging business ventures », Journal of Business Venturing 13 , p. $353-369$.

Chen, MH; Wang, MC (2008). «Social networks and a new venture's innovative capability : the role of trust within entrepreneurial teams », $R \& D$ Management, vol 38, $\mathrm{n}^{\circ} 3$, p. 253-264.

Christensen, C. (1953). Management Succession in Small and Growing Enterprises. Boston : Division of Research, Harvard Business School,

Cooney, T.M. (2005). «What is an Entrepreneurial Team?», International Small Business Journal, Vol. 23, No. 3, pp. 226-235

Cooper, A. C.; Daily, C.M. (1997). «Entrepreneurial team», in D.L. Sexton et R.W. Smilor (eds) Entrepreneurship 2000, pp. 127-50. Chicago, IL : Upstart Publishing.

Creswell, John W. (2009). Research design : Qualitative, quantitative, and mixed methods approaches, London : Sage Publications, Inc.

Cruz, Allan Discual; Howorth, Carole; Hamilton, Eleanor. (2013), «Intrafamily entrepreneurship : the formation and membership of family entrepreneurial teams», Entrepreneurship Theory and Practice, janvier, p. 17-46

De Massis, Alfredo; Chua, Jess H.; Chrisman, James J. (2008). «Factors Preventing Intra-Family Succession», Family Business Review, vol 21, n², p. 183-199. 
Deschamps, Bérangère (2002). «Les spécificités du processus repreneurial », Revue Française de Gestion, $n^{\circ} 138$, avril-juin, p. $175-188$.

Dyck, B.; Mauws, M.; Starke, F. A.,; Mischke, G. A. (2002). «Passing the baton: The importance of sequence, timing, technique, and communication in executive succession », Journal of Business Venturing, vol 17, p. 143-162.

Eisenhardt, Kathleen M.; Schoonhoven Claudia B. (1990). «Organizational Growth : Linking Founding Team, Strategy, Environment and Growth Among U.S. Semiconductor Ventures», Administrative Science Quarterly, vol 35, p. 504-529.

Ensley, Michael D.; Pearson Allison W. (2005), «An exploratory comparison of the behavioral dynamics of top management teams in family and nonfamily new ventures : cohesion, conflict, potency and consensus», Entrepreneurship Theory and Practice, mai, p 267-284.

Ensley, M.D.; Carland, J.W.,; Carland, J.C. (2000). «Investigating the Existence of the Lead Entrepreneur», Journal of Small Business Management, vol 38, n4, p. 59-77.

Farrington, S.M.; Venter, E.; Boshoff, C. (2012). «The role of selected team design elements in successful sibling teams", Family Business Review, vol. 25 n², p. 191-205.

Francis, D.H.; SANDBERG, W.R. (2000), «Friendship within entrepreneurial teams and its association with team and venture performance», Entrepreneurship Theory and Practice vol 25, $n^{\circ} 2, p 5-26$.

Granovetter, M.S. (1985). «Economic action and social structure : the problem of embeddedness », American Journal of Sociology, vol. 91, n³, Novembre, p. 481-510.

GRANT, R. M. (1991). «The resource-based theory of competitive advantage : Implications for strategy formulation», California Management Review, vol. 33, $\mathrm{n}^{\circ} 3, \mathrm{p}$ 114-135.

Handler, Wendy C. (1994). «Succession in Family Business : A Review of the Research», Family Business Review, Vol 7 n²; p. $133-157$

HARPER, D.A. (2008), «Toward a theory of entrepreneurial teams », Journal of Business venturing vol 23, p 613-626.

HARRISON, R.T.; LeITCH, C.M (2005). «Entrepreneurial learning : researching the interface between learning and the entrepreneurial context», Entrepreneurship Theory and Practice vol 29, p. 351-371

Hawkins, Kathryn (2011), «Passing the baton from team to team », Family Business Magazine, Autumn, p 51-53

Huberman, A. M.; Miles, M.B. (1991). Analyse des données qualitatives - Recueil de nouvelles méthodes, Paris, Bruxelles : De Boeck Université

IAcoBucci, D.; Rosa, P. (2010). «The growth of business groups by habitual entrepreneurs : the role of entrepreneurial teams », Entrepreneurship Theory and Practice, Vol $34 n^{\circ} 2$ p 351-377.

Kamm, J.B.; Shuman, J.C.; Seeger J.A.; Nurick, A.J. (1990). «Entrepreneurial teams in new venture creation : a research agenda », Entrepreneurship Theory and Practice, vol 14, ${ }^{\circ} 4$, p. 7-17.

KAMM, J.B.,; NURICK, A.J. (1993). «The stage of team venture formation : A decision-making model », Entrepreneurship Theory and Practice, Winter, p. 17-27.
Kotkin, J. (1986). «The "smart team" at Compaq Computer», INC, $\mathrm{p} 48-56$

LAmbrecht, Johan (2005). «Multigenerational Transition in Family Businesses : A New Explanatory Model», Family Business Review, Vol 18 n4, p. 267-282

Le Breton-Miller, Isabelle; Miller, Danny; Steier, Lloyd P. (2004). "Toward an Integrative Model of Effective FOB Succession», Entrepreneurship Theory and Practice, vol 28 $\mathrm{n}^{\circ} 4$, p. $305-328$

LoBEt, Delphine (2006). «La logique du don dans la transmission des entreprises familiales», Sociétés contemporaines, Vol 2, $\mathrm{n}^{\circ} 62$, p. 27-47.

Lung, Aegean; Foo, Maw Der; Chaturvedi, Sankalp. (2013). «Imprinting Effects of Founding Core Teams on HR Values in New Ventures », Entrepreneurship Theory and Practice, janvier, p 87-105.

Moreau, R (2006). «La formation des équipes d'entrepreneurs », Revue de l'Entrepreneuriat, vol 5 n², p 55-68

Mouline, Jean-Pierre. (2000). «Dynamique de la succession managériale dans la PME familiale non cotée», Finance Contrôle Stratégie, vol 3 n¹, mars, p 197-222.

Naffakhi, H.; Boughattas-Zrig, Y.; Bayad, M. (2008). «La création de connaissances au niveau des équipes entrepreneuriales », Revue de l'entrepreneuriat, vol $7 \mathrm{n}^{\circ} 1$, p. 23-38

Paturel, Robert (2005). «Pistes de réflexion en vue de l'élaboration d'une grille de positionnement des pratiques en Entrepreneuriat», $4^{\mathrm{eme}}$ Congrès de l'Académie de l'Entrepreneuriat, Paris.

Perez-Lizaur, M. (1997). «The Mexican family enterprise faces the open market», Organization, vol 4, p. 535-551

Pfeffer J.; Salancik G. (1978). The external control of organizations, Harper and Row Publishers, New York.

RoYer, Susanne; Simons, Roland; Boyd, Britta; Rafferty, Alannah (2005). «Promoting Family : A Contingency Model of Family Business Succession», Family Business Review, vol 21, p. 15-30.

Sharma, Pramodita (2004). «An Overview of the Field of Family Business Studies : Current Status and Directions for the Future», Family Business Review, vol n¹, p. 1-36

Schjoedt, Leon; Monsen, Erik; Pearson, Allison; Barnett, Tim; Chrisman, James J. (2013). «New venture and family business team : understanding team formation, composition, behaviors, and performance», Entrepreneurship Theory and Practice, janvier, p 1-15

Stake, R.E. (1994). "Case studies», dans N.K. Denzin et Y.S. Lincoln, Handbook of qualitative research, Sage Publications, p 236-247.

STEWART, A. (2003). «Help one another, use one another : Toward an anthropology of family business », Entrepreneurship Theory \& Practice, vol 27, été, p. 383-396

STRAuss, A. (1987). Qualitative research for social scientists, Cambridge : Cambridge University Press.

Timmons, Jeffry (1979). «Careful Self-Analysis and Team Assessment Can Aid Entrepreneurs", Harvard Business Review, vol 57, n6, p. 189-196. 
Ucbasaran, D.; Lockett, A.; Wright, M.; Westhead, P. (2003). «Entrepreneurial Founder Teams : Factors Associated with Team Member Entry and Exit», Entrepreneurship, Theory and Practice, vol 29, p. 107-128.

Ucbasaran, D. (2008). «The Fine Science of Entrepreneurial Decision Making», Journal of Management Studies, vol 45, p. 221-237.

Venter, E; Boshoff, C.; Maas, G. (2005). «The Influence of Successor-Related Factors on the Succession Process in Small and Medium-Sized Family Businesses», Family Business Review, Vol. 18, No. 4, pp. 283-303

WACHEUX, Frédéric. (1996). Méthodes qualitatives et recherche en gestion, Paris, Editions Economica.

West, G. P. (2007). «Collective cognition : When entrepreneurial teams, not individuals, make decisions », Entrepreneurship Theory and Practice, vol 31, p. 77-102.

Westhead, P. (1997). «Ambitions, 'External' Environment and Strategic Factor Differences Between Family and Non-Family Companies», Entrepreneurship and Regional Development, vol $9, n^{\circ} 2$, p. 127-157.

Whyte, M.K. (1996). «The Chinese family and economic development : obstacle or engine?», Economic Development and cultural Change, vol. 44, p. 1-30.

YIN, R. (1994). Case study research : Design and methods (2nd ed.), Beverly Hills, CA : Sage Publishing. 